\title{
PARAMETRIC POINCARÉ-PERRON THEOREM WITH APPLICATIONS
}

\author{
JULIUS BORCEA ${ }^{\dagger}$, SHMUEL FRIEDLAND, AND BORIS SHAPIRO* $^{*}$
}

\begin{abstract}
We prove a parametric generalization of the classical PoincaréPerron theorem on stabilizing recurrence relations where we assume that the varying coefficients of a recurrence depend on auxiliary parameters and converge uniformly in these parameters to their limiting values. As an application we study convergence of the ratios of families of functions satisfying finite recurrence relations with varying functional coefficients. For example, we explicitly describe the asymptotic ratio for two classes of biorthogonal polynomials introduced by Ismail and Masson.
\end{abstract}

\section{INTRODUCTION}

Consider a usual linear recurrence relation of length $k+1$ with constant coefficients

with $\alpha_{k} \neq 0$.

$$
u_{n+1}+\alpha_{1} u_{n}+\alpha_{2} u_{n-1}+\ldots+\alpha_{k} u_{n-k+1}=0
$$

Definition 1. The left-hand side of the equation

$$
t^{k}+\alpha_{1} t^{k-1}+\alpha_{2} t^{k-2}+\ldots+\alpha_{k}=0
$$

is called the characteristic polynomial of recurrence (1.1). Denote the roots of (1.2) by $\lambda_{1}, \ldots, \lambda_{k}$ and call them the spectral numbers of the recurrence.

The following simple theorem can be found in e.g. [20, Ch. 4].

Theorem 1. Let $k \in \mathbb{N}$ and consider a $k$-tuple $\left(\alpha_{1}, \ldots, \alpha_{k}\right)$ of complex numbers with $\alpha_{k} \neq 0$. For any function $u: \mathbb{Z}_{+} \rightarrow \mathbb{C}$ the following conditions are equivalent:

(i) $\sum_{n \geq 0} u_{n} t^{n}=\frac{P(t)}{Q(t)}$, where $Q(t)=1+\alpha_{1} t+\alpha_{2} t^{2}+\ldots+\alpha_{k} t^{k}$ and $P(t)$ is a polynomial in $t$ whose degree is smaller than $k$.

(ii) For all $n \geq k-1$ the function $u_{n}$ satisfies relation (1.1).

(iii) For all $n \geq 0$ one has

$$
u_{n}=\sum_{i=1}^{r} P_{i}(n) \lambda_{i}^{n},
$$

where $\lambda_{1}, \ldots, \lambda_{r}$ are the distinct spectral numbers of (1.1) with multiplicities $m_{1}, \ldots, m_{r}$, respectively, and $P_{i}(n)$ is a polynomial in the variable $n$ of degree at most $m_{i}-1$ for $1 \leq i \leq r$.

The $k$-tuple $\left(u_{0}, \ldots u_{k-1}\right)$ which can be chosen arbitrarily is called the initial $k$-tuple. Denote the $k$-dimensional space of all initial $k$-tuples as $\mathbb{C}^{k}$.

2000 Mathematics Subject Classification. Primary 30C15; Secondary 42C05, 58K15, 58K20.

Key words and phrases. Asymptotic ratio distribution, maxmod-discriminants.

$\dagger$ J. B. unexpectedly passed away on April 8, 2009 at the age of 40. We dedicate this paper (started jointly with J. B. in Spring 2004) to the memory of this talented and tragic human being. Rest in peace, Julius.

* Corresponding author. 
Definition 2. Recurrence relation (1.1) and its characteristic polynomial (1.2) are called maxmod-generic if there exists a unique and simple spectral number $\lambda_{\max }$ of this recurrence satisfying $\left|\lambda_{\max }\right|=\max _{1 \leq i \leq k}\left|\lambda_{i}\right|$. Otherwise (1.1) and (1.2) are called maxmod-nongeneric. The number $\lambda_{\max }$ will be referred to as the leading spectral number of (1.1) or the leading root of (1.2).

Definition 3. An initial $k$-tuple of complex numbers $\left(u_{0}, u_{1}, \ldots, u_{k-1}\right) \in \mathbb{C}^{k}$ is called fast growing with respect to a given maxmod-generic recurrence (1.1) if the coefficient $\kappa_{\max }$ of $\lambda_{\max }^{n}$ in (1.3) is nonvanishing, that is, $u_{n}=\kappa_{\max } \lambda_{\max }^{n}+\ldots$ with $\kappa_{\max } \neq 0$. Otherwise the $k$-tuple $\left(u_{0}, u_{1}, \ldots, u_{k-1}\right)$ is said to be slow growing.

Remark 1 . Note that by Definition 2 the leading spectral number $\lambda_{\max }$ of any maxmod-generic recurrence has multiplicity one. An alternative characterization of fast growing initial $k$-tuples is that they have the property $\lim _{n \rightarrow \infty} \frac{u_{n+1}}{u_{n}}=\lambda_{\text {max }}$. One easily sees that the set of all slowly growing initial $k$-tuples is a (complex) hyperplane in $\mathbb{C}^{k}$, its complement being the set of all fast growing $k$-tuples.The latter hyperplane of slow growing $k$-tuples can be found explicitly using linear algebra.

A famous and frequently used generalization of Theorem 1 in the case of variable coefficients was obtained by H. Poincaré in 1885, [18] and later extended by O. Perron, [17].

Theorem 2 (Poincaré-Perron). If the coefficients $\alpha_{i, n}, \quad i=1, \ldots, k$ of a linear homogeneous difference equation

$$
u_{n+k}+\alpha_{1, n} u_{n+k-1}+\alpha_{2, n} u_{n+k-2}+\ldots+\alpha_{k, n} u_{n}=0
$$

have limits $\lim _{n \rightarrow \infty} \alpha_{i, n}=\alpha_{i}, \quad i=1, \ldots, k$ and if the roots $\lambda_{1}, \ldots, \lambda_{k}$ of the characteristic equation $t^{k}+\alpha_{1} t^{k-1}+\ldots+\alpha_{k}=0$ have distinct absolute values then

(i) for any solution $u$ of (1.4) either $u(n)=0$ for all sufficiently large $n$ or $\lim _{n \rightarrow \infty} \frac{u(n+1)}{u(n)}$ for $n \rightarrow \infty$ equals one of the roots of the characteristic equation.

(ii) if additionally $\alpha_{k, n} \neq 0$ for all $n$ then for every $\lambda_{i}$ there exists a solution $u$ of (1.4) with $\lim _{n \rightarrow \infty} \frac{u(n+1)}{u(n)}=\lambda_{i}$.

Remark 2. If as above $\lambda_{\max }$ will denote the root of the limiting characteristic equation with the maximal absolute value then under the assumptions of Theorem 2 (ii) the set of solutions of (1.4) for which $\lim _{x \rightarrow \infty} \frac{u(n+1)}{u(n)} \neq \lambda_{\max }$ is a complex hyperplane in the space of all solutions. For the latter fact to hold the assumption that all $\lambda_{i}$ 's have distinct absolute values can be substituted by the weaker assumption of maxmod-genericity of the limiting recurrence, see details in Lemma 3. But, in general, there seems to be no easy way to determine this hyperplane explicitly.

A number of generalizations and applications of the Poincaré-Perron theorem can be found in the literature, see e.g. [11], [12], [14], [16], [19] and references therein. The set-up of Poincaré-Perron is often generalized to the case of Poincaré difference systems. Namely, consider an iteration scheme:

$$
\mathbf{u}(n+1)=[A+B(n)] \mathbf{u}(n),
$$

where $\mathbf{u}(n)$ is a vector in $\mathbb{C}^{k}, A$ and $B(n), n=0,1, \ldots$ are $k \times k$-matrices such that $\|B(n)\| \rightarrow 0$ as $n \rightarrow \infty$. For example, one of quite recent results in this direction having a very strong resemblance with Theorem 2 is as follows, see [19], Theorem 1. 
Theorem 3. Let $\mathbf{u}$ be a solution of the system (1.5) under the assumption that $\|B(n)\| \rightarrow 0$ as $n \rightarrow \infty$. Then either $\mathbf{u}(n)=0$ for all sufficiently large $n$ or

$$
\rho=\lim _{n \rightarrow \infty} \sqrt[n]{\|u(n)\|}
$$

exists and is equal to the modulus of one of the eigenvalues of the matrix $A$.

In connection with the present project the second author earlier obtained the following generalization of the Poincare-Perron theorem for the case of Poincaré difference systems, see [5, Theorem 1.2]. This statement apparently covers the majority of results in this direction known at present. Let $\mathrm{M}_{\mathrm{k}}(\mathbb{F})$ and $\mathrm{GL}_{\mathrm{k}}(\mathbb{F})$ denote the spaces of all and resp. of all invertible $(k \times k)$-matrices over a field $\mathbb{F}$.

Theorem 4. Let $\left\{T_{n}\right\}_{n \in \mathbb{N}}$ be a sequence of regular matrices in $\mathrm{GL}_{k}(\mathbb{C})$ converging to some (possibly singular) matrix $T \in \mathrm{M}_{k}(\mathbb{C})$. Assume furthermore that $T$ has a positive spectral radius $\rho(T)$ and that the circle $\{z \in \mathbb{C}|| z \mid=\rho(T)\}$ contains exactly one eigenvalue $\lambda_{\max }$ of $T$ which is a simple root of its characteristic equation. Let $\mathbf{u}_{\max }$ denote an eigenvector of $T$ corresponding to $\lambda_{\max }$, i.e., T $\mathbf{u}_{\max }=\lambda_{\max } \mathbf{u}_{\max }$, $\mathbf{0} \neq \mathbf{u}_{\max } \in \mathbb{C}^{k}$. Then the complex line spanned by the product $T_{n} T_{n-1} \cdots T_{1} \in$ $\mathrm{M}_{k}(\mathbb{C})$ converges to the complex line spanned by $\mathbf{u}_{\max } \mathbf{w}^{t} \in \mathrm{M}_{k}(\mathbb{C})$ for some fixed vector $\mathbf{0} \neq \mathbf{w} \in \mathbb{C}^{k}$. Hence for any vector $\mathbf{x}_{0} \in \mathbb{C}^{k}$ such that $\mathbf{w}^{t} \mathbf{x}_{0} \neq 0$ the complex line in $\mathbb{C}^{k}$ spanned by $T_{n} T_{n-1} \cdots T_{1} \mathbf{x}_{0}$ converges to the complex line in $\mathbb{C}^{k}$ spanned by $\mathbf{u}_{\max }$ as $n \rightarrow \infty$.

Remark 3. Similarly to the situation with Theorem 2 there seems to be no easy way to explicitly determine the vector $\mathbf{w}$ in Theorem 4 .

The goal of the present paper is to present an extension of Theorem 4 for sequences of invertible matrices depending continuously or analytically on auxiliary parameters. In other words, we are looking for a parametric Poincaré-Perron theorem. The main result of the present paper is as follows.

Theorem 5. Let $\left\{T_{n}(\mathbf{x})\right\}_{n \in \mathbb{N}}$ be a sequence of families of regular matrices in $\mathrm{GL}_{k}(\mathbb{C})$ depending continuously on $\mathbf{x} \in \mathcal{D} \subset \mathbb{R}^{d}$. Assume that this sequence converges uniformly, on any compact set in $\mathcal{D}$, to a matrix $T(\mathbf{x})$. Suppose furthermore that for each $\mathbf{x} \in \mathcal{D}, T(\mathbf{x})$ has exactly one simple eigenvalue $\lambda_{\max }(\mathbf{x})$ of the maximal modulus with the corresponding eigenvector $\hat{\mathbf{u}}_{\max }(\mathbf{x}) \in \mathbb{P}^{k-1}$. Then the product $T_{n}(\mathbf{x}) T_{n-1}(\mathbf{x}) \ldots T_{2}(\mathbf{x}) T_{1}(\mathbf{x})$, viewed as an automorphism of $\mathbb{P}^{k-1}$, converges to the transformation $\hat{\mathbf{u}}_{\max }(\mathbf{x}) \hat{\mathbf{w}}^{\mathrm{t}}(\mathbf{x})$ on $\mathbb{P}^{k-1} \backslash \widehat{H(\mathbf{x})}$, where $\hat{\mathbf{w}}(\mathbf{x}) \in \mathbb{P}^{k-1}$ is continuous in $\mathcal{D}$ and $H(\mathbf{x})$ is the hyperplane given by $\mathbf{w}(\mathbf{x})^{\mathrm{t}} \mathbf{v}=0$. (Here $\mathbb{P}^{k-1}$ stands for the complex projective space of dimension $k-1$.)

Remark 4. In Theorem 5 we consider continuous dependence of $\left\{T_{n}(\mathbf{x})\right\}$ on $\mathbf{x}$ and the uniform convergence of this sequence to the limiting $T(\mathbf{x})$. The same result holds in the analytic category if $\left\{T_{n}(\mathbf{x})\right\}$ depend analytically on $\mathbf{x}$ and converge uniformly to $T(\mathbf{x})$. One can also get the same result in the smooth category under the assumption that $\left\{T_{n}(\mathbf{x})\right\}$ depend smoothly on $\mathbf{x}$ and converge uniformly to $T(\mathbf{x})$ together with their partial derivatives of all orders. Since we consider families of complex matrices depending continuously on parameters we decided to use $\mathbb{R}^{d}$ as a parameter space. The case when parameters belong to $\mathbb{C}^{d}$ would be equally natural.

Remark 5. In a sense Theorems 4 and 5 can be considered as a far reaching and parametric generalization of the well-known power method in linear algebra which 
is a simple iterative procedure allowing one to determine the dominating eigenvalue and eigenvector of a given square matrix possessing a unique eigenvalue with maximal modulus, see e.g. [24]. On the other hand, the fact that we consider sequences of matrices (instead of one and the same matrix) additionally depending on extra parameters creates substantial technical difficulties.

In spite of its simple formulation it seems that Theorem 5 has no prototypes in the existing literature. We can now apply Theorems 2, 4 and 5 to sequences of functions satisfying finite linear recurrence relations. The set-up is as follows.

Problem. Given a positive integer $k \geq 2$ let $\left\{\phi_{i, n}(\mathbf{x})\right\}, 1 \leq i \leq k, n \in \mathbb{Z}_{+}$, be a sequence of $k$-tuples of complex-valued functions of a (multi-)variable $\mathbf{x}=$ $\left(x_{1}, \ldots, x_{d}\right)$ defined in some domain $\Omega \subseteq \mathbb{R}^{d}$. Describe the asymptotics when $n \rightarrow \infty$ of the ratio $\Psi_{n}(\mathbf{x})=\frac{f_{n+1}(\mathbf{x})}{f_{n}(\mathbf{x})}$ for a family of complex-valued functions $\left\{f_{n}(\mathbf{x}) \mid n \in\right.$ $\left.\mathbb{Z}_{+}\right\}$satisfying

$$
f_{n+k}(\mathbf{x})+\sum_{i=1}^{k} \phi_{i, n}(\mathbf{x}) f_{n+k-i}(\mathbf{x})=0, \quad n \geq k-1 .
$$

In other words, given a family $\left\{f_{n}(\mathbf{x})\right\}$ of functions satisfying (1.6) calculate the asymptotic ratio $\Psi(\mathbf{x})=\lim _{n \rightarrow \infty} \Psi_{n}(\mathbf{x})$ (if it exists).

To formulate our further results we need some notions. Denote by $\mathrm{Pol}_{k}=$ $\left\{t^{k}+a_{1} t^{k-1}+\ldots+a_{k} \mid a_{i} \in \mathbb{C}, 1 \leq i \leq k\right\}$ the set of all monic polynomials of degree $k$ with complex coefficients.

Definition 4. The subset $\Xi_{k} \subset$ Pol $_{k}$ consisting of all maxmod-nongeneric polynomials is called the standard maxmod-discriminant, see Definition 2. For any family

$$
\Gamma\left(t, \alpha_{1}, \ldots, \alpha_{q}\right)=\left\{t^{k}+a_{1}\left(\alpha_{1}, \ldots, \alpha_{q}\right) t^{k-1}+\ldots+a_{k}\left(\alpha_{1}, \ldots, \alpha_{q}\right)\right\}
$$

of monic polynomials of degree $k$ in $t$ we define the induced maxmod-discriminant $\Xi_{\Gamma}$ to be the set of all parameter values $\left(\alpha_{1}, \ldots, \alpha_{q}\right) \in \mathbb{C}^{q}$ for which the corresponding polynomial in $t$ is maxmod-nongeneric, i.e. belongs to $\Xi_{k}$.

Some local properties of $\Xi_{k}$ can also be derived from [3] and [15].

Example. For $k=2$ the maxmod-discriminant $\Xi_{2} \subset \mathrm{Pol}_{2}$ is the real hypersurface consisting of the set of all pairs $\left(a_{1}, a_{2}\right)$ such that there exists $\epsilon \in[1, \infty)$ solving the equation $\epsilon a_{1}^{2}-4 a_{2}=0$, see Lemma 7 . More information on $\Xi_{k}$ will be given in $\S 6$.

Now take a family

$$
\left\{\bar{\phi}_{n}:=\left(\phi_{1, n}(\mathbf{x}), \phi_{2, n}(\mathbf{x}), \ldots, \phi_{k, n}(\mathbf{x})\right) \mid n \in \mathbb{Z}_{+}\right\}, \mathbf{x}=\left(x_{1}, \ldots, x_{d}\right)
$$

of $k$-tuples of complex-valued functions defined on some domain $\Omega \subset \mathbb{R}^{d}$ such that

(i) $\phi_{k, n}(\mathbf{x})$ is non-vanishing in $\Omega$;

(ii) $\bar{\phi}_{n}$ converges to a fixed $k$-tuple of functions $\widetilde{\phi}=\left(\widetilde{\phi}_{1}(\mathbf{x}), \widetilde{\phi}_{2}(\mathbf{x}), \ldots, \widetilde{\phi}_{k}(\mathbf{x})\right)$ pointwise in $\Omega$.

Choose some initial $k$-tuple of functions $I=\left(f_{0}(\mathbf{x}), \ldots, f_{k-1}(\mathbf{x})\right)$ defined on $\Omega$ and determine the family of functions $\left\{f_{n}(\mathbf{x}) \mid n \in \mathbb{Z}_{+}\right\}$that satisfies the recurrence relation (1.6) for all $n \geq k$ and coincides with $I=\left(f_{0}, \ldots, f_{k-1}\right)$ for $0 \leq n \leq k-1$.

Theorems 2, 4 and 5 imply the following.

Theorem 6. In the above notation there exists a unique subset $\Sigma_{I} \subseteq \Omega \backslash \Xi_{\widetilde{\phi}}$ which is minimal with respect to inclusion and such that the following holds: 
(i) For any $\mathbf{x} \in \Omega \backslash\left(\Xi_{\widetilde{\phi}} \cup \Sigma_{I}\right)$ one has

$$
\lim _{n \rightarrow \infty} \frac{f_{n+1}(\mathbf{x})}{f_{n}(\mathbf{x})}=\Psi_{\max }(\mathbf{x}),
$$

where $\Psi_{\max }(\mathbf{x})$ is the leading root of the asymptotic symbol equation

$$
\Psi^{k}+\widetilde{\phi}_{1}(\mathbf{x}) \Psi^{k-1}+\widetilde{\phi}_{2}(\mathbf{x}) \Psi^{k-2}+\ldots+\widetilde{\phi}_{k}(\mathbf{x})=0
$$

and $\Xi_{\tilde{\phi}}$ denotes the induced maxmod-discriminant of (1.7) considered as a family of monic polynomials in the variable $\Psi$ (cf. Definition 4).

(ii) If $\bar{\phi}_{n}$ consists of continuous functions and $\bar{\phi}_{n} \rightrightarrows \widetilde{\phi}=\left(\widetilde{\phi}_{1}(\mathbf{x}), \widetilde{\phi}_{2}(\mathbf{x}), \ldots, \widetilde{\phi}_{k}(\mathbf{x})\right)$ in $\Omega$ then

$$
\frac{f_{n+1}(\mathbf{x})}{f_{n}(\mathbf{x})} \rightrightarrows \Psi_{\max }(\mathbf{x}) \text { in } \Omega \backslash\left(\Xi_{\tilde{\phi}} \cup \Sigma_{I}\right),
$$

where $\rightrightarrows$ stands for uniform convergence on compact subsets in $\Omega$.

Remark 6. Notice that since we assumed that convergence in (ii) is uniform then each $\widetilde{\phi}_{i}(\mathbf{x})$ is continuous in $\Omega$. Moreover, since a uniformly converging sequence of analytic functions necessarily converges to a analytic function one has that (ii) holds in the analytic category as well. To get the latter result in the smooth category one should require the uniform convergence $\bar{\phi}_{n} \rightrightarrows \widetilde{\phi}$ together with their partial derivatives of all orders.

Definition 5. The set $\Sigma_{I}$ introduced in Theorem 6 is called the set of slowly growing initial conditions (cf. Definition 3). If the functional coefficients in (1.6) are fixed (i.e., independent of $n$ ) then $\Sigma_{I}$ is exactly the set of all points $p \in \Omega$ such that the initial $k$-tuple $I(p)=\left(f_{0}(p), \ldots, f_{k-1}(p)\right)$ is slowly growing with respect to the recurrence (1.6) evaluated at $p$.

Remark 7 . The exact description of $\Sigma_{I}$ in the case of varying coefficients can be found in the proof of Theorem 6 , see $\S 2$. Note that convergence of $\frac{f_{n+1}(\mathbf{x})}{f_{n}(\mathbf{x})}$ to the leading root of (1.7) might actually occur at some points $\mathbf{x}$ lying in $\Xi_{\widetilde{\phi}}$ where the leading root is still unique but multiple.

When both the coefficients $\phi_{i, n}(z)$ and the functions $f_{n}(z)$ are complex analytic one can associate to each meromorphic ratio $\frac{f_{n+1}(z)}{f_{n}(z)}$ the following useful complexvalued distribution $\nu_{n}$, see $[1$, p. 249]. (Since we will only use this distribution in one-dimensional case we will define it for $\mathbb{C}$. For the multi-dimensional case consult e.g. [22].)

Definition 6. Given a meromorphic function $g$ in some open set $\Omega \subseteq \mathbb{C}$ we construct its (complex-valued) residue distribution $\nu_{g}$ as follows. Let $\left\{z_{m} \mid m \in \mathbb{N}\right\}$ be the (finite or infinite) set of all the poles of $g$ in $\Omega$. Assume that the Laurent expansion of $g$ at $z_{m}$ has the form $g(z)=\sum_{-\infty<l \leq l_{m}} \frac{T_{m, l}}{\left(z-z_{m}\right)}$. Then the distribution $\nu_{g}$ is given by

$$
\nu_{g}=\sum_{m \geq 1}\left(\sum_{1 \leq l \leq l_{m}} \frac{(-1)^{l-1}}{(l-1) !} T_{m, l} \frac{\partial^{l-1}}{\partial z^{l-1}} \delta_{z_{m}}\right),
$$

where $\delta_{z_{m}}$ is the Dirac mass at $z_{m}$. The above sum is meaningful as a distribution on $\Omega$ since it is locally finite there.

Remark 8. The distribution $\nu_{g}$ is a complex-valued measure if and only if $g$ has all simple poles, see [1, p. 250]. If the latter holds then in the notation of Definition 6 the value of this complex measure at $z_{m}$ equals $T_{m, 1}$, i.e., the residue of $g$ at $z_{m}$. 
Definition 7. If $\left\{f_{n}(z)\right\}$ consists of functions which are analytic in $\Omega$ and $\nu_{n}$ denotes the residue distribution of the meromorphic function $\frac{f_{n+1}(z)}{f_{n}(z)}$ in $\Omega$ then the limit $\nu=\lim _{n \rightarrow \infty} \nu_{n}$ (if it exists in the sense of weak convergence) is called the asymptotic ratio distribution of the family $\left\{f_{n}(z)\right\}$.

Remark 9. Notice that the support of $\nu$ describes the asymptotics of the zero loci of the family $\left\{f_{n}(z)\right\}$.

Proposition 1. Under the assumptions of Theorem 6 (ii) in the complex analytic category the following holds:

(i) The support of $\nu$ belongs to $\Xi_{\widetilde{\phi}}$, where, as before, $\Xi_{\widetilde{\phi}}$ denotes the induced maxmod-discriminant. The set $\Sigma_{I}$ of slowly growing initial conditions is irrelevant to the support of $\nu$.

(ii) Suppose that there exists a nonisolated point $p_{0} \in \Xi_{\widetilde{\phi}}$ such that equation (1.7) considered at $p_{0}$ has the property that among its roots with maximal absolute value there are at least two with the same maximal multiplicity. If the sequence $\left\{\frac{f_{n+1}\left(p_{0}\right)}{f_{n}\left(p_{0}\right)} \mid n \in \mathbb{Z}_{+}\right\}$diverges then the support of $\nu$ coincides with $\Xi_{\widetilde{\phi}}$.

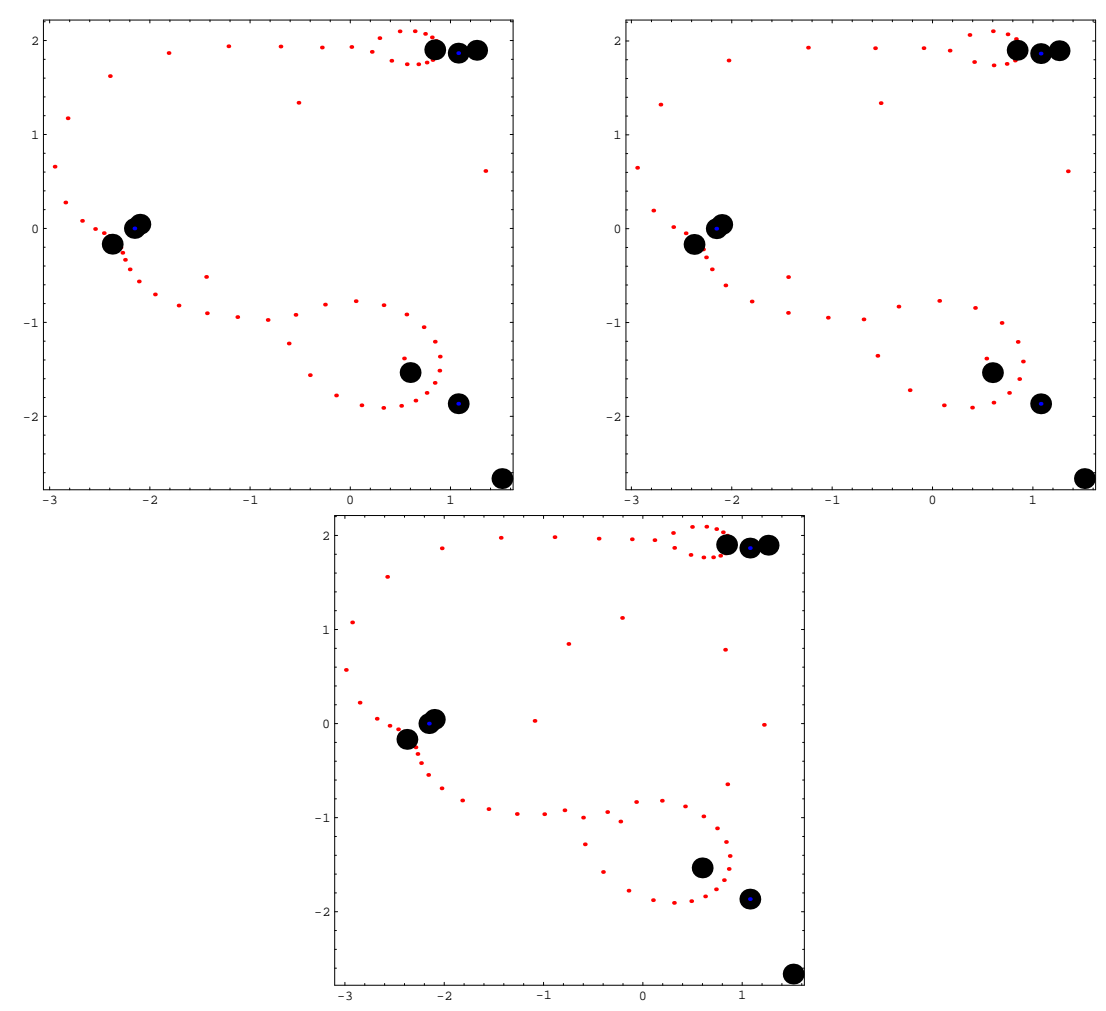

FIGURE 1. Zeros of polynomials satisfying the 4-term recurrence relation $p_{n+1}(z)=p_{n}(z)+(z+1)(z-i) p_{n-1}(z)+\left(z^{3}+10\right) p_{n-2}(z)$

Let us illustrate the latter result in a concrete situation. Consider a sequence of polynomials $\left\{p_{n}(z)\right\}$ satisfying the 4 -term recurrence relation

$$
p_{n+1}(z)=p_{n}(z)+(z+1)(z-i) p_{n-1}(z)+\left(z^{3}+10\right) p_{n-2}(z)
$$


with fixed coefficients and starting with some initial triple of polynomials $I=$ $\left(p_{0}(z), p_{1}(z), p_{2}(z)\right)$. Consider the sequence $\left\{\mathbb{Z}\left(p_{n}\right)\right\}$ of the zero loci of $p_{n}(z)$. Then, one can roughly divide the zeros in $\mathbb{Z}\left(p_{n}\right)$ in 2 parts. The zeros in the first part fill when $n \rightarrow \infty$ the maxmod-discriminant $\Xi_{\widetilde{\phi}} \subset \mathbb{C}$ which is a continuous curve. The second part consisting of finitely many points depends of the initial triple and represents the set $\Sigma_{I}$ of slowly growing conditions.

Explanations to Figure 1. The two upper pictures show the zeros of $p_{64}(z)$ and $p_{45}(z)$ for the same initial triple $p_{0}(z)=0, p_{1}(z)=z^{4}-5 i, p_{2}(z)=z$. The lower picture shows the zeros of $p_{64}(z)$ for the same recurrence relation but with another initial triple $p_{0}(z)=z^{8}-z^{5}+i, p_{1}(z)=z-5 i, p_{2}(z)=5 i z^{2}+z-10$. One observes that on all three pictures the zeros split into 2 parts where the first part forms a pattern close to a smooth curve $\Xi_{\widetilde{\phi}}$ and a the second part consists of a number of isolated points. On the upper pictures there are four isolated points which practically coincide on both pictures although the polynomials themselves are coprime. On the lower picture there are seven isolated points which also form a very stable set, as these points hardly change if one takes different polynomials $p_{n}(z)$ with the same initial triple. The nine fat points in these three pictures are the branching points of the symbol equation $\Psi^{3}=\Psi^{2}+(z+1)(z-i) \Psi+\left(z^{3}+10\right)$.

Remark 10. The isolated points on Figure 1 have a strong resemblance with the spurious poles that were considered in a substantial number of papers on Padé approximation, see e.g. [21] and references therein. The study of the exact relation between these two objects can be found in [2].

This paper is organized as follows. In $\S 2$ we prove our parametric PoincaréPerron theorem and settle the remaining results in $\S 3$. In $\S 4$ we consider concrete examples of 3-term recurrence relations with polynomial coefficients related to the theory of biorthogonal polynomials [10]. In $\S 5$ we discuss a number of related topics and open problems. Finally, in appendix we study the topological structure of the standard maxmod-discriminant $\Xi_{k} \subset \mathrm{Pol}_{k}$.

Acknowledgements. The authors want to thank the anonymous referee for the constructive criticism which allowed us to substantially improve the quality and the clarity of our exposition.

\section{Proving parametric Poincaré-Perron theorem.}

For $\mathbb{F}=\mathbb{R}, \mathbb{C}$ denote by $\mathbb{F}^{k}, M_{k}(\mathbb{F}), \mathrm{GL}_{k}(\mathbb{F})$ the $k$-dimensional vector space, the algebra of $(k \times k)$-matrices and the subgroup of $k \times k$ invertible matrices over the field $\mathbb{F}$. Denote by $\|\cdot\|$ any vector norm on $\mathbb{F}^{k}$ or on $M_{k}(\mathbb{F})$. Let $\|\cdot\|_{2}$ be the $\ell_{2}$-norm on $\mathbb{F}^{k}$ induced by the standard inner product $\langle\mathbf{x}, \mathbf{y}\rangle:=\mathbf{y}^{*} \mathbf{x}$ on $\mathbb{F}^{k}$ and denote by $\|\cdot\|_{2}$ the induced operator norm on $M_{k}(\mathbb{F})$. For $T \in M_{k}(\mathbb{C})$ denote by $\rho(T)$ the spectral radius of $T$, i.e. the maximal modulus of the eigenvalues of $T$. As in [6], the spaces $\mathbb{P F}^{k}, \mathbb{P} M_{k}(\mathbb{F}), \mathbb{P G L}_{k}(\mathbb{F})$ are obtained by identifying the orbits of the action of $\mathbb{F}^{*}:=\mathbb{F} \backslash\{0\}$ on the nonzero elements of the corresponding sets by multiplication. Then $\mathbb{P R}^{k}, \mathbb{P} M_{k}(\mathbb{R})$ and $\mathbb{P} \mathbb{C}^{k}, \mathbb{P} M_{k}(\mathbb{C})$ are compact real and complex manifolds respectively. To keep our notation standard we set $\mathbb{P}^{k-1}=\mathbb{P C}^{k}$. For $\mathbf{x} \in \mathbb{F}^{k} \backslash\{\mathbf{0}\}, T \in M_{k}(\mathbb{F}) \backslash\{0\}$ denote by $\hat{\mathbf{x}}, \hat{T}$ the induced elements in $\mathbb{P F}^{k}, \mathbb{P} M_{k}(\mathbb{F})$ respectively. Furthermore, $\hat{T}$ can be viewed as a map from $\mathbb{P F}^{k} \backslash \widehat{\operatorname{ker} T}$ to $\mathbb{P F}^{k}$. Denote by $d(\cdot, \cdot): \mathbb{P}^{k-1} \times \mathbb{P}^{k-1} \rightarrow[0, \infty)$ the Fubini-Study metric on $\mathbb{P}^{k-1}$, see e.g. [7].

Consider an iteration scheme

$$
\mathbf{x}_{n}:=T_{n} \mathbf{x}_{n-1}, \quad \mathbf{x}_{0} \in \mathbb{F}^{k}, T_{n} \in M_{k}(\mathbb{F}), n \in \mathbb{N} .
$$


This system is called convergent if $\mathbf{x}_{n}, n \in \mathbb{N}$ is a convergent sequence for each $\mathbf{x}_{0} \in$ $\mathbb{F}^{k}$. This is equivalent to the convergence of the infinite product $\ldots T_{n} T_{n-1} \ldots T_{2} T_{1}$, which is defined as the limit of $T_{n} T_{n-1} \ldots T_{2} T_{1}$ as $n \rightarrow \infty$. For the stationary case $T_{n}=T, n \in \mathbb{N}$ the necessary and sufficient conditions for convergency are well known. Namely, (i) the spectral radius $\rho(T)$ can not exceed 1 ; (ii) if $\rho(T)=1$, then 1 is an eigenvalue of $T$ and all its Jordan blocks have size 1; (iii) all other eigenvalues $\lambda$ of $T$ different from 1 satisfy $|\lambda|<1$.

In some instances, as e.g. for problems related to the Lyapunov exponents in dynamical systems, one is interested if the line spanned by the vector $\mathbf{x}_{i}$ converges for all $\mathbf{x}_{0} \neq 0$ in some homogeneous open Zariski set in $\mathbb{F}^{k}$. If this condition holds we call (2.1) projectively convergent.

For the stationary case $0 \neq T_{n}=T \in M_{k}(\mathbb{C})$ one can easily check that $(2.1)$ is projectively convergent if and only if among all the eigenvalues $\lambda$ of $T$ satisfying $|\lambda|=\rho(T)$, there is exactly one eigenvalue $\lambda_{0}$ which has Jordan blocks of the maximal size.

The main point of Theorem 5 is to show that $\hat{\mathbf{w}}(\mathbf{x})$ is continuous in $\mathcal{D}$. We use some technique developed in [6], in particular, the arguments of the proof of Theorem 4, [6, Theorem 1.2, page 258 ], to obtain the continuity of $\hat{\mathbf{w}}(\mathbf{x})$. Namely, let $\lambda_{\max }(\mathbf{x})$ is the unique simple eigenvalue of $T(\mathbf{x})$ with the maximal modulus. Then $\lambda_{\max }(\mathbf{x})$ is continuous and nonvanishing in $\mathcal{D}$. To prove Theorem 5 , we replace $T(\mathbf{x})$ and $T_{n}(\mathbf{x})$ by $\frac{1}{\lambda_{\max }(\mathbf{x})} T(\mathbf{x})$ and $\frac{1}{\lambda_{\max }(\mathbf{x})} T_{n}(\mathbf{x})$ for each $n$, respectively. Thus we can assume that for each $\mathbf{x} \in \mathcal{D}$ the number $\lambda_{\max }=1$ is the simple eigenvalue of $T(\mathbf{x})$ of the maximal modulus. Let $\mathbf{u}(\mathbf{x}), \mathbf{v}(\mathbf{x}), \neq \mathbf{0}$ for each $\mathbf{x} \in \mathcal{D}$, be the right and resp. the left eigenvectors of $T(\mathbf{x})$ corresponding to 1, i.e.

$$
T(\mathbf{x}) \mathbf{u}(\mathbf{x})=\mathbf{u}(\mathbf{x}), \quad T^{\mathrm{t}}(\mathbf{x}) \mathbf{v}(\mathbf{x})=\mathbf{v}(\mathbf{x}), \quad \mathbf{u}^{\mathrm{t}}(\mathbf{x}) \mathbf{v}(\mathbf{x})=1, \quad \mathbf{x} \in \mathcal{D} .
$$

Since $\mathbf{u}(\mathbf{x}), \mathbf{v}(\mathbf{x})$ can be chosen continuous in $\mathcal{D}$ it follows that $\hat{\mathbf{u}}(\mathbf{x}), \hat{\mathbf{v}}(\mathbf{x}) \in \mathbb{P}^{n-1}$ are continuous in $\mathcal{D}$ as well. In what follows we fix a norm $\|\cdot\|$ on $M_{k}(\mathbb{C})$ satisfying for any $T \in M_{k}(\mathbb{C})$ the condition $\|T\|=\left\|T^{\mathrm{t}}\right\|$.

We need two additional Lemmas; the next one generalizes the inequalities given on pages $254-255$ of [6].

Lemma 1. Let $\left\{T_{n}(\mathbf{x})\right\}_{n \in \mathbb{N}}$ be a sequence of complex-valued invertible $(k \times k)$ matrices, with continuous entries for $\mathbf{x} \in \mathcal{D} \subset \mathbb{R}^{d}$. Assume that this sequence converges uniformly, on any compact set in $\mathcal{D}$, to a matrix $T(\mathbf{x})$. Suppose furthermore that for each $\mathbf{x} \in \mathcal{D}$, the number $\lambda_{\max }=1$ is a simple eigenvalue of $T(\mathbf{x})$, and all other eigenvalues of $T(\mathbf{x})$ lie in the open unit disk $|\lambda|<1$. Then for any fixed $\mathbf{x}_{0}$ and for each $\varepsilon>0$ there exists $\delta=\delta(\varepsilon)>0$ and $N=N(\varepsilon), m=m(\varepsilon) \in \mathbb{N}$ such that

$$
\begin{array}{r}
\left\|T_{n+m}(\mathbf{x}) T_{n+m-1}(\mathbf{x}) \ldots T_{n+1}(\mathbf{x})-\mathbf{u}\left(\mathbf{x}_{0}\right) \mathbf{v}^{\mathrm{t}}\left(\mathbf{x}_{0}\right)\right\|<\varepsilon \\
\text { for each } n \geq N \text { and }\left|\mathbf{x}-\mathbf{x}_{0}\right| \leq \delta .
\end{array}
$$

Proof. Since 1 a simple eigenvalue of $T\left(\mathbf{x}_{0}\right)$ and all other eigenvalues of $T\left(\mathbf{x}_{0}\right)$ lie in the open unit disk, it follows that $\lim _{m \rightarrow \infty} T^{m}\left(\mathbf{x}_{0}\right)=\mathbf{u}\left(\mathbf{x}_{0}\right) \mathbf{v}^{\mathrm{t}}\left(\mathbf{x}_{0}\right)$. Hence, there exists $m=m(\varepsilon)$ so that $\left\|T^{m}\left(\mathbf{x}_{0}\right)-\mathbf{u}\left(\mathbf{x}_{0}\right) \mathbf{v}^{\mathrm{t}}\left(\mathbf{x}_{0}\right)\right\|<\frac{\varepsilon}{2}$. Since the product map $\left(M_{k}(\mathbb{C})\right)^{m} \rightarrow M_{k}(\mathbb{C})$ sending a $m$-tuple $X_{1}, \ldots, X_{m}$ to their product is continuous, there exists $\delta_{1}>0$, such that for $\left\|X_{i}-T\left(\mathbf{x}_{0}\right)\right\| \leq \delta_{1}$ for $i=1, \ldots, m$ the inequality $\left\|X_{1} X_{2} \ldots X_{m}-T\left(\mathbf{x}_{0}\right)^{m}\right\| \leq \frac{\varepsilon}{2}$ holds. Since $T_{n}(\mathbf{x})$ converges uniformly to the continuous $T(\mathbf{x})$ on any compact subset of $\mathcal{D}$, it follows that there exists $\delta=\delta(\varepsilon)>0$ and $N=N(\varepsilon) \in \mathbb{N}$ such that $\left\|T_{n}(\mathbf{x})-T\left(\mathbf{x}_{0}\right)\right\| \leq \frac{\varepsilon}{2}$ for $n \geq N$ and $\left\|\mathbf{x}-\mathbf{x}_{0}\right\| \leq \delta$.

Next we give the following modification of [6, Lemma 5.1]. 
Lemma 2. Let $E \in M_{k}(\mathbb{C})$ be a matrix of rank one with $\rho(E)>0$, i.e. $E=$ $\mathbf{v u}^{\mathrm{t}}, \mathbf{u}^{\mathrm{t}} \mathbf{v}=1$. Set $O_{r}:=\left\{\hat{\mathbf{x}} \in \mathbb{P} \mathbb{C}^{k}: d(\hat{\mathbf{x}}, \hat{\mathbf{v}}) \leq r\right\}$ such that $O_{r} \cap \widehat{\operatorname{ker} E}=\emptyset$. Then $\hat{E}: O_{r} \rightarrow\{\hat{\mathbf{v}}\}$. Then there exists $\varepsilon=\varepsilon(r)$ such that the following conditions hold. Assume that $B \in M_{k}(\mathbb{C})$ satisfies $\|B-E\| \leq \varepsilon$. Then

(1) $d(\hat{B} \hat{\mathbf{x}}, \hat{\mathbf{v}}) \leq \frac{r}{2}$ for each $\hat{\mathbf{x}} \in \mathbb{O}_{r}$.

(2) $d(\hat{B} \hat{\mathbf{x}}, \hat{B} \hat{\mathbf{y}}) \leq \frac{1}{2} d(\hat{\mathbf{x}}, \hat{\mathbf{y}})$ for each $\hat{\mathbf{x}}, \hat{\mathbf{y}} \in O_{r}$.

Proof. Clearly $\widehat{E \mathbf{x}}=\hat{\mathbf{v}}$ if $\mathbf{u}^{\mathrm{t}} \mathbf{x} \neq 0$. Hence $\hat{E}: O_{r} \rightarrow\{\hat{\mathbf{v}}\}$. Recall that for any $B \in M_{k}(\mathbb{C}) \backslash\{0\}$ the transformation $\hat{B}: \mathbb{P} \mathbb{C}^{k} \backslash \widehat{\operatorname{ker} B} \rightarrow \mathbb{P} \mathbb{C}^{k}$ is analytic. Hence there exists $\varepsilon_{0}>0$ so that for $\|B-E\| \leq \varepsilon_{0}, B \neq 0$, and $\widehat{\operatorname{ker} B} \cap O_{r}=\emptyset$. Thus, $\hat{B}$ is analytic on $O_{r}$. One has that for a small $\epsilon \in\left(0, \epsilon_{0}\right)$ any $\hat{B}$ satisfying $\|B-E\| \leq \varepsilon$ is a small perturbation of $\hat{E}$. Since $\hat{E} O_{r}=\{\hat{\mathbf{v}}\}$ we deduce condition 1 for any $\varepsilon \in\left(0, \varepsilon_{1}\right)$ for some $0<\varepsilon_{1}<\varepsilon_{0}$. Since $d(\hat{E} \hat{\mathbf{x}}, \hat{E} \hat{\mathbf{y}})=0$ for any $\hat{\mathbf{x}}, \hat{\mathbf{y}} \in O_{r}$ we deduce condition 2 for some $\varepsilon \in\left(0, \varepsilon_{1}\right)$.

Proof of Theorem 5. Recall that [6, Theorem 1.2] implies that the map $\widehat{T_{n}(\mathbf{x})} \widehat{T_{n-1}(\mathbf{x})} \ldots \widehat{T_{2}(\mathbf{x})} \widehat{T_{1}(\mathbf{x})}$, viewed as an automorphism of $\mathbb{P}^{k-1}$, converges pointwise to the transformation $\hat{\mathbf{u}}(\mathbf{x}) \hat{\mathbf{w}}^{\mathrm{t}}(\mathbf{x})$ on $\mathbb{P}^{k-1} \backslash H(\mathbf{x})$. Now fix some $\mathbf{x}_{0} \in \mathcal{D}$. Then there exists $\delta_{1}>0$ such that the eigenvectors $\mathbf{u}(\mathbf{x}), \mathbf{v}(x) \in \mathbb{C}^{k}$ satisfying (2.2) can be chosen continuously in $\left|\mathbf{x}-\mathbf{x}_{0}\right| \leq \delta_{1}$. Since $\mathbf{v}^{\mathrm{t}}(\mathbf{x}) \mathbf{u}(x)=1$ it follows that we can choose $\delta_{2} \in\left(0, \delta_{1}\right)$ such that $\mathbf{v}\left(\mathbf{x}_{0}\right) \mathbf{u}(\mathbf{x}) \neq 0$ for $\left|\mathbf{x}-\mathbf{x}_{0}\right| \leq \delta_{1}$. To show the continuity of $\hat{\mathbf{w}}(\mathbf{x})$ in $\left|\mathbf{x}-\mathbf{x}_{0}\right|<\delta$ for some $\delta_{3} \in\left(0, \delta_{2}\right)$ it is enough to show that the continuity of the limit of $\widehat{\mathbf{v}^{\mathrm{t}}\left(\mathbf{x}_{0}\right)} \widehat{T_{n}(\mathbf{x})} \widehat{T_{n-1}(\mathbf{x})} \ldots \widehat{T_{2}(\mathbf{x})} \widehat{T_{1}(\mathbf{x})}$ in $\left|\mathbf{x}-\mathbf{x}_{0}\right|<\delta_{3}$. Since each $T_{j}(\mathbf{x}) \in \mathrm{GL}(k, \mathbb{C})$ is continuous, it is enough to show the continuity of the limit of $\widehat{\mathbf{v}^{\mathrm{t}}\left(\mathbf{x}_{0}\right)} \widehat{T_{n+N}(\mathbf{x})} \widehat{T_{n+N-1}}(\mathbf{x}) \ldots \widehat{T_{N+2}(\mathbf{x})} \widehat{T_{N+1}(\mathbf{x})}$ in $\left|\mathbf{x}-\mathbf{x}_{0}\right|<\delta_{3}$. One

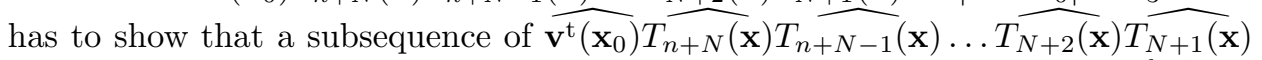
converges uniformly in $\left|\mathbf{x}-\mathbf{x}_{0}\right| \leq \delta_{3}$ in the Fubini-Study metric $d(\cdot, \cdot)$ on $\mathbb{P}^{k-1}$.

This is done by using Lemmas 1-2. Namely, let $E=\mathbf{v}\left(\mathbf{x}_{0}\right) \mathbf{u}^{\mathrm{t}}\left(\mathbf{x}_{0}\right)$. Choose $r>0, \varepsilon>0$ so that the conditions 1-2 of Lemma 2 hold. Next choose $N, \delta$ so that the condition $(2.3)$ holds. Let $\delta_{3}=\min \left(\delta, \delta_{2}\right)$. Define

$$
B_{j}(\mathbf{x})=T_{(j-1) m+N+1}^{\mathrm{t}}(\mathbf{x}) \ldots T_{j m+N}^{\mathrm{t}}(\mathbf{x}) \quad \text { for } j=1, \ldots
$$

Assume that $\left|\mathbf{x}-\mathbf{x}_{0}\right| \leq \delta_{3}$. We claim that the sequence $\widehat{B_{1}(\mathbf{x})} \ldots \widehat{B_{l}(\mathbf{x})} \widehat{\mathbf{v}\left(\mathbf{x}_{0}\right)}$ converges uniformly to $\mathbf{w}(\mathbf{x}) \in O_{r}$ in the Fubini-Study metric. First observe that (2.3) yields that $\widehat{B_{j}(\mathbf{x})} O_{r} \subset O_{\frac{r}{2}}$, by condition 1 of Lemma 2. Hence $\hat{\mathbf{y}}_{l}(\mathbf{x})=$ $\widehat{B_{1}(\mathbf{x})} \ldots \widehat{B_{l}(\mathbf{x})} \widehat{\mathbf{v}\left(\mathbf{x}_{0}\right)} \subset O_{\frac{r}{2}}$. Condition 1 of Lemma 2 yields that

$$
\begin{aligned}
& d\left(\widehat{B_{1}(\mathbf{x})} \ldots \widehat{B_{l}(\mathbf{x})} \widehat{\mathbf{v}\left(\mathbf{x}_{0}\right)}, \widehat{B_{1}(\mathbf{x})} \ldots \widehat{B_{l+p}(\mathbf{x})} \widehat{\mathbf{v}\left(\mathbf{x}_{0}\right)}\right) \leq \\
& \leq \frac{1}{2^{l}} d\left(\widehat{\mathbf{v}\left(\mathbf{x}_{0}\right)}, \widehat{B_{l+1}(\mathbf{x})} \ldots \widehat{B_{l+p}(\mathbf{x})} \widehat{\mathbf{v}\left(\mathbf{x}_{0}\right)}\right) \leq \frac{l}{2^{l}} .
\end{aligned}
$$

Hence the sequence $\hat{\mathbf{y}}_{l}, l \in \mathbb{N}$ converges uniformly in $\left|\mathbf{x}-\mathbf{x}_{0}\right| \leq \delta_{3}$ to $\hat{\mathbf{y}}(\mathbf{x})$. Since each $\hat{\mathbf{y}}_{l}(\mathbf{x})$ is continuous in $\left|\mathbf{x}-\mathbf{x}_{0}\right| \leq \delta_{3}$ it follows that $\mathbf{y}(\mathbf{x})$ is continuous in the open disk $\left|\mathbf{x}-\mathbf{x}_{0}\right|<\delta_{3}$.

\section{Proving Remaining Results}

Consider a recurrence relation with varying coefficients of the form

$$
u_{n+k}+\alpha_{1, n} u_{n+k-1}+\alpha_{2, n} u_{n+k-2}+\ldots+\alpha_{k, n} u_{n}=0
$$


where $\alpha_{k, n} \neq 0$. Assume that for all $i \in\{1, \ldots, k\}$ the one has $\lim _{n \rightarrow \infty} \alpha_{i, n}=: \alpha_{i}$ and denote the limiting recurrence relation of (3.1) by

$$
v_{n+k}+\alpha_{1} v_{n+k-1}+\alpha_{2} v_{n+k-2}+\ldots+\alpha_{k} v_{n}=0 .
$$

Lemma 3. If (3.2) is maxmod-generic then $\lim _{n \rightarrow \infty} \frac{v_{n+1}}{v_{n}}$ exists and equals $\lambda_{\max }$ on the complement of a complex hyperplane $H \subset \mathbb{C}^{k}$, where $\lambda_{\max }$ denotes the leading spectral number of (3.2).

Proof. The result is an immediate corollary of Theorem 4 applied to the family of linear operators $\left\{T_{n} \mid n \in \mathbb{N}\right\}$ whose action on $\mathbb{C}^{k+1}$ is given by

$$
T_{n}=\left(\begin{array}{cccccc}
1 & \alpha_{1, n} & \alpha_{2, n} & \alpha_{3, n} & \ldots & \alpha_{k, n} \\
1 & 0 & 0 & 0 & \ldots & 0 \\
0 & 1 & 0 & 0 & \ldots & 0 \\
\vdots & \vdots & \vdots & \vdots & \ddots & \vdots \\
0 & 0 & 0 & \ldots & 1 & 0
\end{array}\right), \quad n \in \mathbb{N} .
$$

Notice that $\alpha_{k, n} \neq 0$.

The exceptional complex hyperplane $H$ is called the hyperplane of slow growth. From Theorem 4 we deduce the following corollary (cf. [5, Proposition 2.1]):

Corollary 1. Under the assumptions of Theorem 4 there exists a sequence of real numbers $\left\{\theta_{n}\right\}_{n \in \mathbb{N}}$ such that

$$
\lim _{n \rightarrow \infty} e^{i \theta_{n}} \frac{T_{n} T_{n-1} \cdots T_{1}}{\left\|T_{n} T_{n-1} \cdots T_{1}\right\|}=\mathbf{u}_{\max } \mathbf{w}^{t}
$$

the convergence taking place in $\mathrm{M}_{k}(\mathbb{C})$.

We now settle Theorem 6 .

Proof. [Theorem 6 (i)] Let us first determine $\Sigma_{I}$ and at the same time prove the pointwise convergence of $\frac{f_{n+1}\left(x_{1}, \ldots, x_{d}\right)}{f_{n}\left(x_{1}, \ldots, x_{d}\right)}$ to $\Psi_{\max }\left(x_{1}, \ldots, x_{d}\right)$ in the complement $\Omega \backslash\left(\Xi_{\tilde{\phi}} \cup \Sigma_{I}\right)$. One can view the set-up of Theorem 6 as the situation when the recurrence relation (3.1) depends on additional parameters $\mathbf{x}=\left(x_{1}, \ldots, x_{d}\right)$. Thus if at a given point $p \in \Omega \subseteq \mathbb{R}^{d}$ the $\operatorname{limit}_{\lim _{n \rightarrow \infty}} \frac{f_{n+1}(p)}{f_{n}(p)}$ does not exist then $p$ lies in the induced maxmod-discriminant $\Xi_{\widetilde{\phi}}$ (cf. Definition 4). On the other hand, if $\lim _{n \rightarrow \infty} \frac{f_{n+1}(p)}{f_{n}(p)}$ exists but is not equal to $\Psi_{\max }(p)$ and, additionally, $p$ lies in $\Omega \backslash \Xi_{\tilde{\phi}}$ then the corresponding initial $k$-tuple $I(p)=\left(f_{0}(p), \ldots, f_{k-1}(p)\right)$ belongs to the hyperplane $H(p)$ of slow growth at the given point $p$, see Lemma 3 . The latter set of points $p$ is by definition the set $\Sigma_{I}$ of slowly growing initial conditions. For varying coefficients the hyperplane of slow growth at a given point $p \in \Omega \backslash \Xi_{\widetilde{\phi}}$ is determined by $\lim _{n \rightarrow \infty} e^{i \theta_{n}} \frac{T_{n} T_{n-1} \cdots T_{1}}{\left\|T_{n} T_{n-1} \cdots T_{1}\right\|}$, see Corollary 1 . Indeed, Theorem 4 implies that in this case the hyperplane of slow growth $H(p)$ at $p$ consists of all vectors $\mathbf{x}(p) \in \mathbb{C}^{k}$ such that $\mathbf{w}^{t}(p) \mathbf{x}(p)=0$.

To settle Theorem 6 (ii) we assume that $\bar{\phi}_{n} \rightrightarrows \widetilde{\phi}=\left(\tilde{\phi}_{1}, \tilde{\phi}_{2}, \ldots, \tilde{\phi}_{k}\right)$ and all considered functions are continuous in $\Omega$. Let $p_{0} \in \Omega \backslash\left(\Xi_{\widetilde{\phi}} \cup \Sigma_{I}\right)$. From Corollary 1 and the arguments of $[5, \S 4$ and $\S 5]$ it follows that one can find a sufficiently small neighborhood $\mathcal{O}_{p_{0}} \subset \Omega \backslash\left(\Xi_{\tilde{\phi}} \cup \Sigma_{I}\right)$ of $p_{0}$ such that for any $\epsilon>0$ there exists $N_{\epsilon} \in \mathbb{N}$ satisfying

$$
\left\|e^{i \theta_{n}(p)} \frac{K_{n}(p)}{\left\|K_{n}(p)\right\|}-\mathbf{u}_{\max }(p) \mathbf{w}^{t}(p)\right\| \leq \epsilon \text { for } p \in \mathcal{O}_{p_{0}}, n \geq N_{\epsilon},
$$

where $K_{n}(p):=T_{n}(p) T_{n-1}(p) \cdots T_{1}(p)$. Clearly, $\mathbf{u}_{\max }(p) \mathbf{w}^{t}(p)$ is a rank one matrix and so it has precisely one simple eigenvalue of maximum modulus for all 
$p \in \mathcal{O}_{p_{0}}$. From (3.3) we deduce that there exists $N \in \mathbb{N}$ such that the polynomial $\operatorname{det}\left(\lambda I_{k}-K_{n}(p)\right)$ has precisely one simple eigenvalue $\lambda_{\max , n}(p)$ of maximum modulus for all $p \in \mathcal{O}_{p_{0}}$ and $n \geq N$. The implicit function theorem implies that $\lambda_{\max , n}(p)$ is continuous in $\mathcal{O}_{p_{0}}$ for all $n \geq N$, so that $\left\{\frac{K_{n}(p)}{\lambda_{\max , n}(p)}\right\}_{n \geq N}$ is a sequence of matrix-valued functions which are continuous in $\mathcal{O}_{p_{0}}$. On the other hand, it is not difficult to see that this sequence converges pointwise in $\mathcal{O}_{p_{0}}$ to $\mathbf{u}_{\max }(p) \mathbf{w}^{t}(p)$ (see e.g. the proof of Theorem 4 given in [5]). Together with (3.3) this implies that $\left\{\frac{\left\|K_{n}(p)\right\|}{\left|\lambda_{\max , n}(p)\right|}\right\}_{n \geq N}$ is a sequence of (continuous) functions that converges pointwise to the constant function 1 on $\mathcal{O}_{p_{0}}$. It follows that there exists some open subset $\mathcal{O}_{p_{0}}^{\prime} \subset \mathcal{O}_{p_{0}}$ with $p_{0} \in \mathcal{O}_{p_{0}}^{\prime}$ such that $\left\{\frac{\left\|K_{n}(p)\right\|}{\left|\lambda_{\max , n}(p)\right|}\right\}_{n \geq N}$ is a uniformly bounded sequence of functions on $\mathcal{O}_{p_{0}}^{\prime}$. Thus $\left\{\frac{K_{n}(p)}{\lambda_{\max , n}(p)}\right\}_{n \geq N}$ is a bounded sequence of continuous matrix-valued functions which converges pointwise to the function $\mathbf{u}_{\max }(p) \mathbf{w}^{t}(p)$ in $\mathcal{O}_{p_{0}}^{\prime}$. Invoking Vitali's theorem we conclude that

$$
\frac{K_{n}(p)}{\lambda_{\max , n}(p)} \rightrightarrows \mathbf{u}_{\max }(p) \mathbf{w}^{t}(p) \text { in } \mathcal{O}_{p_{0}}^{\prime}
$$

Notation 1. In notation of Theorem 6 let $V=\Omega \times \mathbb{C}^{k}$ denote the Cartesian product of $\Omega$ and the linear space $\mathbb{C}^{k}$ of all initial $k$-tuples at a point in $\Omega$. Any initial $k$-tuple $I=\left(f_{0}(\mathbf{x}), \ldots, f_{k-1}(\mathbf{x})\right)$ of smooth (respectively, analytic) functions in $\Omega$ can be considered as a smooth (respectively, analytic) section of $V$ viewed as a trivial vector bundle over $\Omega$. Denote by $\widetilde{V}$ the restriction of the bundle $V$ to the subset $\Omega \backslash \Xi_{\widetilde{\phi}}$ of the base.

Definition 8. Let $\Delta_{\phi}$ denote the subset of $\widetilde{V}$ consisting of all pairs $(p, I(p))$ such that the asymptotic ratio of the recurrence (1.6) evaluated at $p$ with initial $k$-tuple $I(p) \in \mathbb{C}^{k}$ does not coincide with the leading root of (1.7) at $p$. The set $\Delta_{\phi}$ is called the subvariety of slow growth.

Corollary 2. In the above notation the subvariety $\Delta_{\phi}$ of slow growth is a smooth (resp. an analytic) subbundle of complex hyperplanes in $\widetilde{V}$ provided that the family of $k$-tuples

$$
\left\{\bar{\phi}_{n}=\left(\phi_{1, n}(\mathbf{x}), \phi_{2, n}(\mathbf{x}), \ldots, \phi_{k, n}(\mathbf{x})\right) \mid n \in \mathbb{Z}_{+}\right\}
$$

with $\phi_{k, n}(\mathbf{x})$ non-vanishing in $\Omega$ and its limit $k$-tuple $\widetilde{\phi}=\left(\widetilde{\phi}_{1}(\mathbf{x}), \widetilde{\phi}_{2}(\mathbf{x}), \ldots, \widetilde{\phi}_{k}(\mathbf{x})\right)$ consist of smooth (resp. analytic) functions that satisfy $\bar{\phi}_{n} \rightrightarrows \widetilde{\phi}$ in $\Omega$ in the corresponding category.

Proof. Indeed, by Theorem 6 (ii) the family of hyperplanes of slow growth depend smoothly resp. analytically on $p \in \Omega \backslash \Xi_{\widetilde{\phi}}$ in the corresponding category.

Definition 9. Let $\xi: E \rightarrow B$ be a (complex) vector bundle over a base $B$. A smooth (respectively, analytic) section $S: B \rightarrow E$ is called transversal to a given smooth (respectively, analytic) submanifold $\mathcal{H} \subset E$ if at each point $p$ of the intersection $S \cap \mathcal{H}$ the sum of the tangent spaces at $p$ to $S$ and $\mathcal{H}$ coincides with the tangent space at $p$ to the ambient space $E$. A subset $X$ of a topological space $Y$ is called massive if $X$ can be represented as the intersection of at most countably many open dense subsets in $Y$. 
Remark 11. Thom's transversality theorem, see e.g. [8], implies that the set of all smooth sections of the bundle $\xi: E \rightarrow B$ which are transversal to a given smooth subbundle $\mathcal{H} \subset E$ is a massive subset of the set of all sections. The same holds in the analytic category provided that the fibration is trivial.

Lemma 4. If an initial $k$-tuple $I=\left(f_{0}(\mathbf{x}), \ldots, f_{k-1}(\mathbf{x})\right)$ of smooth (respectively, analytic) functions in $\Omega$ is transversal to the subvariety $\Delta_{\phi}$ of slow growth then the set $\Sigma_{I}$ of slowly growing initial conditions is either empty or a smooth (respectively, analytic) subvariety in $\Omega$ of real codimension two.

Proof. By Thom's transversality theorem the set of all smooth initial $k$ tuples which are transversal to $\Delta_{\Phi}$ is a massive subset of the set of all possible smooth initial $k$-tuples (cf. Remark 11). Combined with Proposition 2 this implies, in particular, that for a given initial $k$-tuple $I$ transversal to $\Delta_{\phi}$ one has that $I$ (considered as a section of $\widetilde{V}$ ) and $\Delta_{\Phi}$ are smooth submanifolds of $\widetilde{V}$ transversal to each other, where $\widetilde{V}$ is the restriction of the bundle $V=\Omega \times \mathbb{C}^{k}$ to the subset $\Omega \backslash \Xi_{\widetilde{\phi}}$ of the base (cf. Notation 1). This transversality property implies that the intersection $I \cap \Delta_{\phi}$ is either empty or a smooth submanifold of $\widetilde{V}$ of real codimension 2. Notice that the image $\xi\left(I \cap \Delta_{\phi}\right)$ of the projection $\xi: \widetilde{V} \rightarrow \Omega \backslash \Xi_{\widetilde{\phi}}$ to the base is exactly $\Sigma_{I}$. Actually, $\xi$ induces a diffeomorphism between $I \cap \Delta_{\phi}$ and $\Sigma_{I}$. To see this one simply notes that $\xi$ maps the section $I$ diffeomorphically to $\Omega \backslash \Xi_{\widetilde{\phi}}$ and that the intersection $I \cap \Delta_{\phi}$ is a smooth submanifold of the section $I$. The proof of the corresponding result in the analytic category is analogous.

In particular, in the one-dimensional case one has the following.

Lemma 5. If the coefficients $\left(\phi_{1, n}(z), \ldots, \phi_{k, n}(z)\right), n \in \mathbb{Z}_{+}$, and the initial $k$-tuple $I=\left(f_{0}(z), \ldots, f_{k-1}(z)\right)$ of recurrence (1.6) are complex analytic functions in an open set $\Omega \subseteq \mathbb{C}$, and $\phi_{k, n}(z) \neq 0$ in $\Omega$ then either $\Xi_{\widetilde{\phi}}=\Omega$ or $\Xi_{\widetilde{\phi}}$ is a union of real analytic curves while $\Sigma_{I}$ is either the whole $\Omega$ or consists of isolated points.

Proof. By Corollary 2 the set $\Delta_{\Phi}$ is analytic in the analytic category. Thus in this case the asymptotic symbol equation (1.7) is either maxmod-nongeneric everywhere in $\Omega \subseteq \mathbb{C}$ or is maxmod-nongeneric on a one-dimensional real analytic subset, which proves the statement in part (i) concerning $\Xi_{\widetilde{\phi}}$. Note that, in fact, in the analytic category $\Xi_{\widetilde{\phi}}$ is always a real semianalytic set and $\Sigma_{I}$ is analytic. Therefore, $\Sigma_{I}$ is either a complex analytic curve, in which case it coincides with $\Omega$, or an analytic zero-dimensional subset of $\Omega$, i.e., the union of isolated points.

We finally turn to Proposition 1 . To settle it we need an additional lemma. which a simple consequence of Theorem 1.

Notation 2. Let $R e c_{k}$ be the $k$-dimensional complex linear space consisting of all $(k+1)$-term recurrence relations with constant coefficients of the form (1.1). As before we denote by $\mathbb{C}^{k}$ the $k$-dimensional complex linear space of all initial $k$-tuples $\left(u_{0}, \ldots, u_{k-1}\right)$.

Definition 10. A maxmod-nongeneric recurrence relation in $R e c_{k}$ with initial $k$-tuple $i n_{k} \in \mathbb{C}^{k}$ is said to be of dominant type if the following conditions are satisfied. Let $\lambda_{1}, \ldots, \lambda_{r}, r \leq k$, denote all distinct spectral numbers with maximal absolute value and assume that these have multiplicities $m_{1}, \ldots, m_{r}$, respectively. Then there exists a unique index $i_{0} \in\{1, \ldots, r\}$ such that $m_{i}<m_{i_{0}}$ for $i \in$ $\{1, \ldots, r\} \backslash\left\{i_{0}\right\}$ and the initial $k$-tuple $i n_{k}$ is fast growing in the sense that the degree of the polynomial $P_{i_{0}}$ in (1.3) corresponding to $\lambda_{i_{0}}$ is precisely $m_{i_{0}}-1$. The number $\lambda_{i_{0}}$ is called the dominant spectral number of this recurrence relation.

Lemma 6. In the above notation the following is true: 
(i) The set of all slowly growing initial $k$-tuples with respect to a given maxmodgeneric recurrence relation in $R_{e} c_{k}$ is a complex hyperplane $\mathcal{S G}_{k}$ in $\mathbb{C}^{k}$. The set $\mathcal{S G}_{k}$ is called the hyperplane of slow growth.

(ii) For any maxmod-generic recurrence relation in $R e c_{k}$ and any fast growing initial $k$-tuple $\left(u_{0}, \ldots, u_{k-1}\right)$ the limit $\lim _{n \rightarrow \infty} \frac{u_{n+1}}{u_{n}}$ exists and coincides with the leading spectral number $\lambda_{\max }$, that is, the (unique) root of the characteristic equation (1.2) with maximal absolute value.

(iii) Given a maxmod-nongeneric recurrence relation of dominant type in Rec $_{k}$ the limit $\lim _{n \rightarrow \infty} \frac{u_{n+1}}{u_{n}}$ exists and coincides with the dominant spectral number.

(iv) For any maxmod-nongeneric recurrence relation of nondominant type in Rec $_{k}$ the set of initial $k$-tuples for which $\lim _{n \rightarrow \infty} \frac{u_{n+1}}{u_{n}}$ exists is a union of complex subspaces of $\mathbb{C}^{k}$ of positive codimensions. This union is called the exceptional variety.

Proof. In order to prove (i) notice that the coefficient $\kappa_{\max }$ in Definition 3 is a nontrivial linear combination of the entries of the initial $k$-tuple $\left(u_{0}, \ldots, u_{k-1}\right)$ with coefficients depending on $\alpha_{1}, \ldots, \alpha_{k}$. Therefore, the condition $\kappa_{\max }=0$ determines a complex hyperplane $\mathcal{S G}_{k}$ in $\mathbb{C}^{k}$. One can easily see that the hyperplane of slow growth is the direct sum of all Jordan blocks corresponding to the spectral numbers of a given recurrence (1.1) other than the leading one.

The assumptions of part (ii) together with (1.3) yield $u_{n}=\kappa_{\max } \lambda_{\max }^{n}+\ldots$ for $n \in \mathbb{Z}_{+}$, where the dots stand for the remaining terms in (1.3) corresponding to the spectral numbers whose absolute values are strictly smaller than $\left|\lambda_{\text {max }}\right|$. Therefore, the quotient $\frac{u_{n+1}}{u_{n}}$ has a limit as $n \rightarrow \infty$ and this limit coincides with $\lambda_{\max }$, as required. By definition $\lambda_{\max }$ is a root of (1.2), which completes the proof of (ii). This last step can alternatively be carried out by dividing both sides of (1.1) by $u_{n-k+1}$ and then letting $n \rightarrow \infty$. In view of Definition 10 the same arguments show that the assertion in (iii) is true as well.

For the proof of part (iv) we proceed as follows. Take any maxmod-nongeneric recurrence relation of the form (1.1) and let $\lambda_{1}, \ldots, \lambda_{r}, r \leq k$, be all its distinct spectral numbers with maximal absolute value. Thus $\left|\lambda_{i}\right|=\left|\lambda_{\max }\right|$ if and only $1 \leq$ $i \leq r$. Choose an initial $k$-tuple $I T=\left(u_{0}, \ldots, u_{k-1}\right)$ and denote by $P_{1}, \ldots, P_{r}$ the polynomials in (1.3) corresponding to $\lambda_{1}, \ldots, \lambda_{r}$, respectively, for the sequence $\left\{u_{n} \mid\right.$ $\left.n \in \mathbb{Z}_{+}\right\}$constructed using the given recurrence with initial $k$-tuple $I T$ as above. Assuming, as we may, that our recurrence relation is nontrivial we get from (1.3) that $\lambda_{i} \neq 0$ if $1 \leq i \leq r$. We may further assume that the degrees $d_{1}, \ldots, d_{r}$ of the polynomials $P_{1}, \ldots, \bar{P}_{r}$, respectively, satisfy $d_{1} \geq \ldots \geq d_{r}$. Under these conditions we now prove that $\lim _{n \rightarrow \infty} \frac{u_{n+1}}{u_{n}}$ exists if and only if exactly one of the polynomials $P_{1}, \ldots, P_{r}$ is nonvanishing. A direct check analogous to the proof of part (ii) shows that if only the polynomial $P_{1}$ is nonvanishing then $\lim _{n \rightarrow \infty} \frac{u_{n+1}}{u_{n}}=\lambda_{1}$. If $r \geq 2$ and $s \in\{2, \ldots, r\}$ is such that $P_{1}, \ldots, P_{s}$ are all nonvanishing polynomials among $P_{1}, \ldots, P_{r}$ then using again $(1.3)$ we get

$$
\frac{u_{n+1}}{u_{n}}=\frac{P_{1}(n+1)+P_{2}(n+1)\left(\frac{\lambda_{2}}{\lambda_{1}}\right)^{n+1}+\ldots+P_{s}(n+1)\left(\frac{\lambda_{s}}{\lambda_{1}}\right)^{n+1}+o(1)}{P_{1}(n)+P_{2}(n)\left(\frac{\lambda_{2}}{\lambda_{1}}\right)^{n}+\ldots+P_{s}(n)\left(\frac{\lambda_{s}}{\lambda_{1}}\right)^{n}+o(1)} .
$$

Since $\left|\frac{\lambda_{i}}{\lambda_{1}}\right|=1$ and $\lambda_{i} \neq \lambda_{1}, 2 \leq i \leq s$, it follows that if $d_{1}=d_{2}$ then the expression in the right-hand side has no limit as $n \rightarrow \infty$. Therefore, if such a limit exists then $d_{1}>d_{2}$, which gives us a complex subspace of $\mathbb{C}^{k}$ of (positive) codimension equal 
to $d_{1}-d_{2}$. Thus the exceptional variety is a union of complex subspaces of $\mathbb{C}^{k}$ of (in general) different codimensions.

Now we prove Proposition 1.

Proof. Under the assumptions of the Proposition 1 the leading root of the asymptotic symbol equation (1.7) is a well-defined analytic function in a sufficiently small neighborhood of $\Sigma_{I}$. Therefore, the residue distribution (1.8) associated to this leading root vanishes in a neighborhood of $\Sigma_{I}$. Thus the set $\Sigma_{I}$ of slowly growing initial conditions can be deleted from the support of the asymptotic ratio distribution $\nu$, which proves (i).

In order to prove that under the nondegeneracy assumptions of (ii) the support of $\nu$ coincides with $\Xi_{\widetilde{\phi}}$ we show that in this case the sequence $\frac{f_{n+1}(p)}{f_{n}(p)}$ diverges for almost all $p \in \Xi_{\widetilde{\phi}}$. Indeed, due to analyticity the first condition of (ii) implies that the recurrence relation is of nondominant type almost everywhere in $\Xi_{\widetilde{\phi}}$. The second condition of (ii) then implies that the sequence $\frac{f_{n+1}(p)}{f_{n}(p)}$ diverges almost everywhere in $\Xi_{\widetilde{\phi}}$, which settles part (ii) of the proposition.

\section{Application to Biorthogonal polynomials}

Here we calculate explicitly the induced maxmod-discriminant $\Xi_{\tilde{\phi}}$ and the asymptotic ratio distribution $\nu$ in a number of cases. We illustrate the relation of $\Xi_{\widetilde{\phi}}$ and $\Sigma_{I}$ with the roots of polynomials satisfying certain recurrence relations. Let us first recall some definitions from [10]. In op. cit. the authors introduced two types of polynomial families related to what they call $R_{I^{-}}$and $R_{I I^{-}}$-type continued fractions, respectively. These families were later studied in [25].

A polynomial family of type $R_{I}$ is a system of monic polynomials generated by

$$
p_{n+1}(z)=\left(z-c_{n+1}\right) p_{n}(z)-\lambda_{n+1}\left(z-a_{n+1}\right) p_{n-1}(z)
$$

with $p_{-1}(z)=0, p_{0}(z)=1$ and $p_{n}\left(a_{n+1}\right) \lambda_{n+1} \neq 0$ for $n \in \mathbb{Z}_{+}$.

A polynomial family of type $R_{I I}$ is a system of monic polynomials generated by

$$
p_{n+1}(z)=\left(z-c_{n+1}\right) p_{n}(z)-\lambda_{n+1}\left(z-a_{n+1}\right)\left(z-b_{n+1}\right) p_{n-1}(z)
$$

with $p_{-1}(z)=0, p_{0}(z)=1$ and $p_{n}\left(a_{n+1}\right) p_{n}\left(b_{n+1}\right) \lambda_{n+1} \neq 0$ for $n \in \mathbb{Z}_{+}$.

Assuming that

$$
\lim _{n \rightarrow \infty} a_{n}=A, \lim _{n \rightarrow \infty} b_{n}=B, \lim _{n \rightarrow \infty} c_{n}=C \text { and } \lim _{n \rightarrow \infty} \lambda_{n}=\Lambda,
$$

where $A, B, C, \Lambda$ are complex numbers, we describe the asymptotic ratio $\Psi_{\max }(z)=$ $\lim _{n \rightarrow \infty} \frac{p_{n+1}(z)}{p_{n}(z)}$ and the asymptotic ratio measure $\nu$ for $R_{I^{-}}$and $R_{I I^{-}}$-type polynomial families. Using our previous results we restrict ourselves to a recurrence relation of the form

$$
p_{n+1}(z)=Q_{1}(z) p_{n}(z)+Q_{2}(z) p_{n-1}(z),
$$

with the initial triple $p_{-1}(z)=0, p_{0}(z)=1, \operatorname{deg} Q_{1}(z)=1$ and $\operatorname{deg} Q_{2}(z) \leq 2$. By Theorem 6 and Corollary 5 the asymptotic ratio $\Psi_{\max }(z)$ satisfies the symbol equation

$$
\Psi_{\max }^{2}(z)=Q_{1}(z) \Psi_{\max }(z)+Q_{2}(z)
$$

everywhere in the complement of the union of the induced maxmod-discriminant $\Xi_{\tilde{\phi}}$ and a finite set of (isolated) points. Notice also that the asymptotic ratio distribution $\nu$ satisfies the relation

$$
\nu=\frac{\partial \Psi_{\max }(z)}{\partial \bar{z}},
$$

where the r.h.s. is interpreted as a distribution. By our previous results the support of $\nu$ coincides with $\Xi_{\widetilde{\phi}}$. In order to describe $\Xi_{\widetilde{\phi}}$ we need the following simple lemma. 


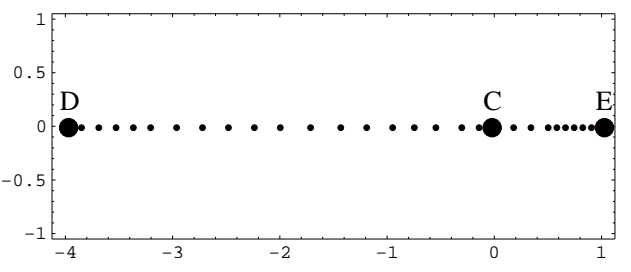

FIGURE 2. Zeros of $p_{41}(z)$ satisfying the 3 -term recurrence relation $p_{n+1}(z)=z p_{n}(z)+\left(\frac{3 z-4}{4}\right) p_{n-1}(z)$ with $p_{-1}(z)=0$ and $p_{0}(z)=1$

Lemma 7. A quadratic polynomial $t^{2}+a_{1} t+a_{2}$ has two roots with the same absolute value if and only if there exists a real number $\epsilon \in[1, \infty)$ such that $\epsilon a_{1}^{2}-4 a_{2}=0$.

Proof. The roots of the polynomial are given by $t_{1,2}=\frac{-a_{1} \pm \sqrt{a_{1}^{2}-4 a_{2}}}{2}$, so that if $\left|t_{1}\right|=\left|t_{2}\right|$ then the complex numbers $a_{1}$ and $\sqrt{a_{1}^{2}-4 a_{2}}$ considered as vectors in $\mathbb{C}$ must be orthogonal. Thus $\left|\operatorname{Arg}\left(a_{1}\right)-\operatorname{Arg} \sqrt{a_{1}^{2}-4 a_{2}}\right|=\frac{\pi}{2}$, which is equivalent to saying that $\frac{\sqrt{a_{1}^{2}-4 a_{2}}}{a_{1}}$ is purely imaginary and therefore $\frac{a_{1}^{2}-4 a_{2}}{a_{1}^{2}} \in(-\infty, 0]$. The converse statement is obvious.

Corollary 3. The induced maxmod-discriminant $\Xi_{\tilde{\phi}} \subset \mathbb{C}$ is the set of all solutions $z$ to the equation

where $\epsilon \in[1, \infty)$.

$$
\epsilon Q_{1}^{2}(z)+4 Q_{2}(z)=0
$$

We start with the situation when $Q_{1}(z)$ and $Q_{2}(z)$ are real polynomials. Let $C$ denote the unique (real) root of $Q_{1}(z)$ and denote by $D, E$ the branching points of the symbol equation, i.e., the roots of $Q_{1}^{2}(z)+4 Q_{2}(z)=0$. If $Q_{1}(z)$ and $Q_{2}(z)$ are real there are three basic cases:

(1) $D$ and $E$ are real, $D<E$ and $C \in[D, E]$;

(2) $D$ and $E$ are complex conjugate;

(3) $D$ and $E$ are real, $D<E$ and $C \notin[D, E]$.

Case 1. The discriminant $\Xi_{\widetilde{\phi}}$ is the interval $[D, E]$. The density $\rho_{\nu}$ of $\nu$ in this case is positive and equals

$$
\rho_{\nu}(x)=\frac{i}{2 \pi} \sqrt{Q_{1}^{2}(x)+4 Q_{2}(x)} d x,
$$

where the value of $\sqrt{Q_{1}^{2}(x)+4 Q_{2}(x)}$ is taken with negative imaginary part. (The value of $Q_{1}^{2}(x)+4 Q_{2}(x)$ on the interval $[D, E]$ is negative.)

Remark 12. Note that $\int_{D}^{E} d \nu$ is not necessarily equal to 1 . In fact, $\int_{D}^{E} d \nu=\frac{(E-D)^{2}}{16}$. For comparison let us take the sequence of inverse ratios $\frac{p_{n}(z)}{p_{n+1}(z)}$ for the above sequence of monic polynomials $\left\{p_{n}(z)\right\}$. Let $\tilde{\nu}_{n}$ denote the residue distribution of $\frac{p_{n}(z)}{p_{n+1}(z)}$ and set $\tilde{\nu}=\lim _{n \rightarrow \infty} \tilde{\nu}_{n}$. One can easily see that the distribution $\tilde{\nu}$ is actually a probability measure (i.e., it satisfies $\int_{D}^{E} d \widetilde{\nu}=1$ ) having the same support as $\nu$ and its density $\rho_{\widetilde{\nu}}$ is given by

$$
\rho_{\widetilde{\nu}}(x)=-\frac{i}{2 \pi} \frac{\sqrt{Q_{1}^{2}(x)+4 Q_{2}(x)}}{Q_{2}(x)} d x .
$$

For the concrete example depicted in Figure 2 the asymptotic ratio measure $\nu$ equals $\frac{2 \sqrt{-x^{2}-3 x+4}}{25 \pi} d x$ and is supported on the interval $[-4,1]$. 


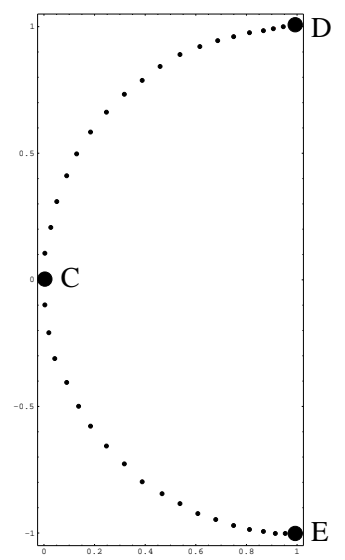

FIGURE 3 . Zeros of $p_{41}(z)$ satisfying the 3 -term recurrence relation $p_{n+1}(z)=z p_{n}(z)+\left(\frac{1-z}{2}\right) p_{n-1}(z)$ with $p_{-1}(z)=0$ and $p_{0}(z)=1$

Case 2. The discriminant $\Xi_{\tilde{\phi}}$ is the arc $D-C-E$ of the unique circle passing through all three points $D, C, E$. In order to simplify the notation while calculating $\nu$ assume that $\operatorname{deg} Q_{1}(z)=1$. Then by an affine change of the variable $z$ we can normalize $Q_{1}(z)$ such that $Q_{1}(z)=z$. Set $Q_{2}(z)=a z^{2}+b z+c$, where $a, b, c$ are real numbers. The condition that $Q_{1}^{2}(z)+4 Q_{2}(z)=0$ has complex conjugate roots gives $b^{2}-c(4 a+1)<0$. Then the center of the circle passing through $D, C, E$ is given by $\left(-\frac{c}{b}, 0\right)$ and its radius is $\left|\frac{c}{b}\right|$. If $\gamma$ is the angle from the real positive half-axis to the ray through the center of the circle and a point $(x, y)$ on it then we have the following parametrizations: $x=\left|\frac{c}{b}\right| \cos \gamma-\frac{c}{b}, y=\left|\frac{c}{b}\right| \sin \gamma$ and $n=\cos \gamma+i \sin \gamma$ for the unit normal to the circle at the point $(x, y)$.

In order to calculate the asymptotic ratio measure $\nu=\frac{\partial \Psi_{\max }(z)}{\partial \bar{z}}$ notice that if $\Psi_{\max }(z)$ is a piecewise analytic function with smooth curves separating the domains where $\Psi_{\max }(z)$ coincides with a given analytic function then $\nu=\frac{\partial \Psi_{\max }(z)}{\partial \bar{z}}$ is concentrated on these separation curves. Additionally, at each smooth point where two branches $\Psi_{1}$ and $\Psi_{2}$ meet $\nu$ satisfies the condition $\nu=\frac{\left(\Psi_{1}-\Psi_{2}\right) n d s}{2 \pi}$ (with an appropriate choice of coorientation). Applying now this result to the case under consideration we see that

$$
\nu=\frac{\partial \Psi_{\max }(z)}{\partial \bar{z}}=\frac{\sqrt{Q_{1}^{2}(z)+4 Q_{2}(z)} n}{2 \pi}\left|\frac{c}{b}\right| d \gamma .
$$

Explicit computations (using Mathematica ${ }^{\mathrm{TM}}$ ) give

$$
\nu=\frac{\sqrt{2}}{b}(\cos \gamma+i \sin \gamma)^{\frac{3}{2}} \sqrt{c\left(2 b^{2}-(1+4 a) c(1-\cos \gamma)\right)} d \gamma
$$

For the concrete example considered in Figure 3 one has

$$
\nu=\sqrt{2 \cos \gamma}(\cos \gamma+i \sin \gamma)^{\frac{3}{2}} d \gamma,
$$

where $\gamma$ is the angle from the real positive half-axis to the ray emanating from $\left(-\frac{c}{b}, 0\right)=(1,0)$ (i.e., the center of the circle of radius $\left|\frac{c}{b}\right|=1$ containing the points $D, C, E$ in this case) and passing through a variable point $(x, y)$ lying on the left half-circle.

Case 3. The discriminant $\Xi_{\widetilde{\phi}}$ is the union of the interval $[D, E]$ and the circle given (as in Case 2) by the equation $x\left(x-x_{0}\right)+y^{2}=0$ where $x$ (respectively, $y$ ) is the real (respectively, imaginary) part of $z$ and $x_{0}=-\frac{2 c}{b}$. The density $\rho_{\nu}$ of 
$\nu=\frac{\partial \Psi_{\max }(z)}{\partial \bar{z}}$ is given by formula (4.3) on the interval and by formula (4.4) on the circle. A concrete example is depicted in Figure 4 below.

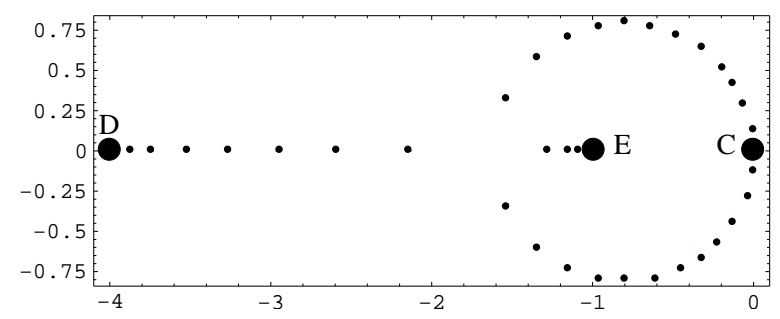

FIgURE 4 . Zeros of $p_{41}(z)$ satisfying the 3 -term recurrence relation $p_{n+1}(z)=z p_{n}(z)+\left(\frac{5 z+4}{4}\right) p_{n-1}(z)$ with $p_{-1}(z)=0$ and $p_{0}(z)=1$

If either of the polynomials $Q_{1}(z)$ and $Q_{2}(z)$ has complex coefficients it seems difficult to give a more precise description of the support of $\nu$ than the one obtained in Corollary 3. Figure 5 below illustrates possible forms of this support.
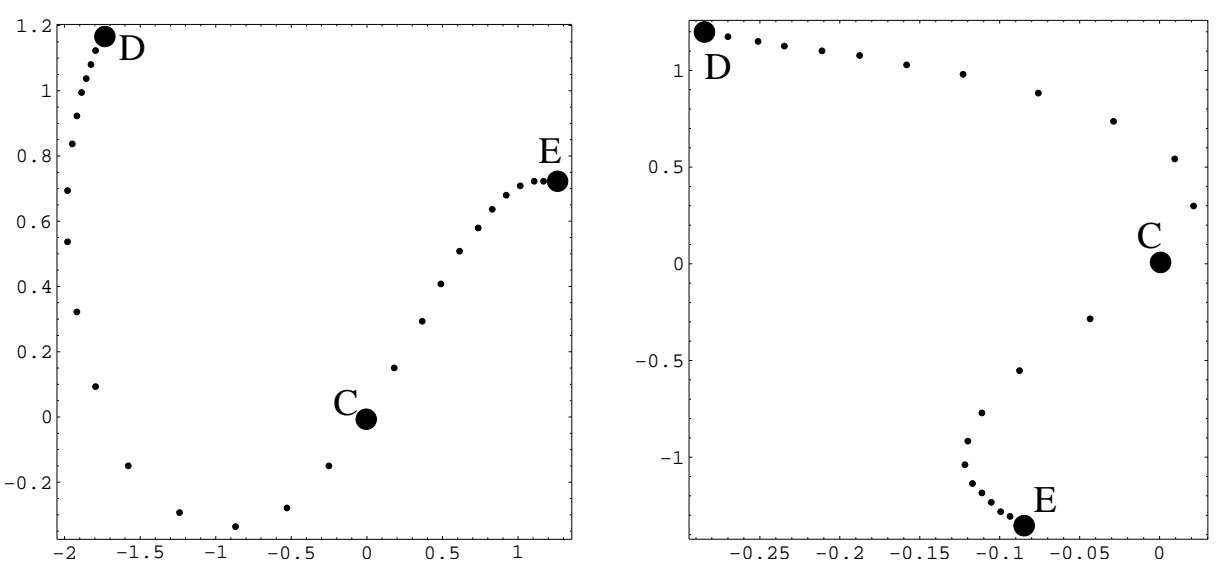

FiguRE 5. Zeros of $p_{41}(z)$ for the recurrence relations $p_{n+1}(z)=$ $z p_{n}(z)+\left(i z^{2}+2 z-1-3 i\right) p_{n-1}(z)$ (left) and $p_{n+1}(z)=z p_{n}(z)+$ $\left((2-i) z^{2}+z+4-i\right) p_{n-1}(z)$ (right) with $p_{-1}(z)=0$ and $p_{0}(z)=1$

Note that in all examples in this section we took the standard initial polynomials $p_{-1}(z)=0$ and $p_{0}(z)=1$. Computer experiments show no isolated zeros of $p_{n}(z)$ in these cases. The following lemma shows that in the specific situation when $p_{-1}(z)=$ 0 and $p_{0}(z)=1$ the set $\Sigma_{I}$ of slowly growing initial conditions (cf. Definition 3 ) is actually empty.

Lemma 8. For any recurrence relation of the form (4.1) with initial values $p_{-1}(z)=$ 0 and $p_{0}(z)=1$ the set $\Sigma_{I}$ of slowly growing initial conditions is empty, comp. [2].

Proof. A recurrence relation of the form (4.1) may be rewritten as

$$
\left(\begin{array}{c}
p_{n+1}(z) \\
p_{n}(z)
\end{array}\right)=T(z)\left(\begin{array}{c}
p_{n}(z) \\
p_{n-1}(z)
\end{array}\right), \text { where } T(z)=\left(\begin{array}{cc}
Q_{1}(z) & Q_{2}(z) \\
1 & 0
\end{array}\right) .
$$

It is easily checked that for any $z \in \mathbb{C}$ the vector $(1,0)^{t} \in \mathbb{C}^{2}$ is not an eigenvector of $T(z)$, which immediately implies the desired result.

We should point out that as soon as one changes the initial pair $\left(p_{-1}(z), p_{0}(z)\right)$ the polynomials $p_{n}(z)$ immediately acquire additional isolated zeros away from $\Xi_{\widetilde{\phi}}$. 


\section{RELATED TOPICS AND OPEN PROBLEMS}

5.1. Does Theorem 5(ii) hold in the algebraic category, i.e. when the varying coefficients are algebraic functions of bounded degree?

5.2. Although one of the assumptions of Theorem 6 is that all $\phi_{k, n}(\mathbf{x})$ are nonvanishing in $\Omega$ computer experiments show that it holds in many cases where some $\phi_{k, n}(\mathbf{x})$ vanish. Apparently one can construct complicated counterexamples when the statement fails, in general. But it would be important for applications to find some sufficient conditions weaker than $\phi_{k, n}(\mathbf{x}) \neq 0$ guaranteeing the validity of Theorem 6(ii).

5.3. Theory of multiple orthogonal polynomials which experiences a rapid development at present is a natural area of application our results, see e.g. [4] and reference therein. Above we only considered a rather simple instance of biorthogonal polynomials. It would be nice to calculate the maxmod-discriminant $\Xi_{\tilde{\phi}}$ and the asymptotic ratio distribution in more examples.

5.4. It is natural to ask what topological information about the induced maxmoddiscriminant may be obtained from the coefficients of a given recurrence relation.

Problem 1. Describe the topological properties of $\Xi_{\widetilde{\phi}}$ depending on the coefficients of the recurrence relation (say assumed fixed and algebraic) and establish, in particular, necessary and sufficient conditions for the set $\Xi_{\widetilde{\phi}}$ to be compact. Are there any characteristic numbers associated with the singularities on $\Xi_{\widetilde{\phi}}$ ?

\section{Appendix. On TOPOLOGY AND GEOMETRY OF MAXMOD-DISCRIMINANTS}

Definition 11. Denote by $\widetilde{\Xi}_{k} \subset \operatorname{Pol}_{k}=\left\{t^{k}+a_{1} t^{k-1}+\ldots+a_{k}\right\}$ the set of all monic polynomials of degree $k$ with complex coefficients having at least two roots with the same absolute value.

It is obvious from Definitions 2,4 and 11 that $\widetilde{\Xi}_{k}$ contains the standard maxmoddiscriminant $\Xi_{k}$.

Proposition 2. $\widetilde{\Xi}_{k}$ is a real semialgebraic hypersurface of degree at most $(4 k-$ 1) $(4 k-2)$ in Pol $_{k}$. Furthermore, the hypersurface $\widetilde{\Xi}_{k}$ is quasihomogeneous with quasihomogeneous weight equal to $i$ for both the real and the imaginary parts of $a_{i}$, $i=1,2, \ldots, k$. (Here Pol $_{k}$ is considered as a $2 k$-dimensional real affine space with the real and imaginary parts of all $a_{i}$ 's chosen as coordinates.)

Proof. In order to calculate the degree of $\widetilde{\Xi}_{k}$ let us describe an algorithm giving the equation for the analytic continuation of $\widetilde{\Xi}_{k}$. This algorithm may be presented as the superposition of two resultants. Let $u=x+i y \in \mathbb{C}$ and consider first the resultant of $P(t)=t^{k}+a_{1} t^{k-1}+\ldots+a_{k}$ and $P(u t)=(u t)^{k}+a_{1}(u t)^{k-1}+\ldots+a_{k}$, which we denote by $R\left(u, a_{1}, \ldots, a_{k}\right)$. (Recall that all $a_{i}$ 's are complex.) Clearly, $R\left(u, a_{1}, \ldots, a_{k}\right)$ is a polynomial in the variable $u$. For any fixed value of $u$ the resultant $R\left(u, a_{1}, \ldots, a_{k}\right)$ vanishes if and only if $P(t)$ and $P(u t)$ considered as polynomials in $t$ have a common zero. One can easily see that $R\left(u, a_{1}, \ldots, a_{k}\right)$ is divisible by $(u-1)^{k}$ and that the quotient $\widetilde{R}\left(u, a_{1}, \ldots, a_{k}\right)=R\left(u, a_{1}, \ldots, a_{k}\right) /(u-1)^{k}$ is coprime with $u-1$. We want to find an equation for the set of all monic complex polynomials $P(t)$ such that $P(u t)$ and $P(t)$ have a common zero for some $u \neq 1$ with $|u|=1$. The standard rational parametrization of the unit circle is given by $u=\frac{(1-i \theta)^{2}}{1+\theta^{2}}$, where $\theta \in \mathbb{R}$. The result of the substitution $u=x+i y=\frac{(1-i \theta)^{2}}{1+\theta^{2}}$ in $\widetilde{R}\left(u, a_{1}, \ldots, a_{k}\right)$ gives a complex-valued rational function of the real variable $\theta$ whose denominator equals to $\left(1+\theta^{2}\right)^{2 k}$. By taking the resultant of the real and imaginary parts of the 
numerator of the latter rational function one gets the required algebraic equation for the analytic continuation of $\widetilde{\Xi}_{k}$. This recipe allows us to calculate (an upper bound for) the degree of $\widetilde{\Xi}_{k}$. It is easy to see that $\widetilde{R}\left(u, a_{1}, \ldots, a_{k}\right)$ is a polynomial of degree $2 k$ in $u$ and of degree at most $2 k-1$ in the variables $a_{1}, \ldots, a_{k}$. After making the substitution $u=x+i y=\frac{(1-i \theta)^{2}}{1+\theta^{2}}$ and taking the real and imaginary parts of the above rational function one gets two polynomials of degrees at most $2 k-1$ in the variables $\Re a_{1}, \ldots, \Re a_{k}, \Im a_{1}, \ldots, \Im a_{k}$ and of degrees $4 k$ and $4 k-1$, respectively, in $\theta$. These polynomials have proportional leading terms and, therefore, their resultant is a polynomial in the variables $\Re a_{1}, \ldots, \Re a_{k}, \Im a_{1}, \ldots, \Im a_{k}$ of degree at most $(2(4 k)-2)(2 k-1)=(4 k-1)(4 k-2)$.

The quasihomogeneity of $\widetilde{\Xi}_{k}$ with quasihomogeneous weights as specified in the statement of the proposition follows from the fact that $\widetilde{\Xi}_{k}$ is preserved if one multiplies all roots of a polynomial by a non-negative real number.

Remark 13. The proof of Proposition 2 contains an algorithm giving the explicit equation for the analytic continuation of $\widetilde{\Xi}_{k}$. The authors wrote a Mathematica ${ }^{\mathrm{TM}}$ code doing this for small values of $k$. However, the resulting expression contains several hundred terms even for $k=3$ and does not seem to be of much use. For $k=3$ the estimate $(4 k-1)(4 k-2)$ for the degree is sharp. It is very plausible that this estimate is actually sharp for arbitrary $k$ although in the general case it seems very difficult to check the necessary nondegeneracy conditions while performing the superposition of the two resultants described in the above algorithm.

Proposition 3. $\widetilde{\Xi}_{k}$ is a singular real hypersurface with a (singular) boundary coinciding with the usual discriminant $\mathcal{D}_{k} \subset$ Pol $_{k}$, where $\mathcal{D}_{k}$ is the set of all polynomials in Pol $_{k}$ with multiple roots. Moreover, $\widetilde{\Xi}_{k}$ has no local singularities (i.e., singularities on a given branch) outside $\mathcal{D}_{k}$. The list of singularities of $\widetilde{\Xi}_{k}$ is finite for any given $k \in \mathbb{N}$, i.e. its singularities have no moduli.

Proof. Indeed, to show that $\mathcal{D}_{k} \subset \operatorname{Pol}_{k}$ is the boundary of $\widetilde{\Xi}_{k}$ consider the standard Vieta map $V i: \mathbb{C}^{k} \rightarrow$ Pol $_{k}$ sending a $k$-tuple of (labeled) roots to the coefficients of the monic polynomial with these roots, i.e, to the elementary symmetric functions of these roots with alternating signs. It is known that the Vieta map induces a local diffeomorphism on the complement $\mathbb{C}^{k} \backslash \mathcal{T}_{k}$, where $\mathcal{T}_{k}$ is the standard Coxeter hyperplane arrangement consisting of $\left(\begin{array}{l}k \\ 2\end{array}\right)$ hyperplanes $L_{i, j}$ given by $L_{i, j}: x_{i}=x_{j}, 1 \leq i<j \leq k$. An easy observation is that $\mathcal{T}_{k}$ coincides with the preimage $V i^{-1}\left(\mathcal{D}_{k}\right)$ of the discriminant $\mathcal{D}_{k}$. Consider now the arrangement of quadratic cones $\mathfrak{C}_{k}=\cup_{i<j} \mathfrak{C}_{i, j}$ in $\mathbb{C}^{k}$, where $\mathfrak{C}_{i, j}$ is given by the equation $\left|x_{i}\right|=\left|x_{j}\right|$. Obviously, $\mathfrak{C}_{k}$ coincides with the preimage $V i^{-1}\left(\widetilde{\Xi}_{k}\right)$. From the defining equation it is clear that each $\mathfrak{C}_{i, j}$ is smooth outside the origin. Therefore, $\widetilde{\Xi}_{k}$ is locally smooth (that is, it consists of smooth local branches) outside $\mathcal{D}_{k}$. Note also that for $1 \leq i<j \leq k$ the quadratic cone $\mathfrak{C}_{i, j}$ contains the complex hyperplane $L_{i, j}$ and that the restriction $\left.V i\right|_{\mathfrak{C}_{i, j}}$ has a fold near a generic point of $L_{i, j}$. Therefore, $\mathcal{D}_{k}$ is the boundary of $\widetilde{\Xi}_{k}$. The absence of the moduli for the singularities of $\widetilde{\Xi}_{k}$ can be derived from that for the singularities of $\mathcal{D}_{k}$. The type of a (multi)singularity of $\widetilde{\Xi}_{k}$ near a polynomial $P(z) \in \widetilde{\Xi}_{k}$ is encoded in the following combinatorial information about the roots of $P(z)$. Determine first the multiplicity (possibly vanishing) of the root of $P(z)$ at the origin and the set of all distinct positive absolute values for all the roots of $P(z)$. For each such positive absolute value determine the number and multiplicity of distinct roots having this absolute value. If all the roots are simple then (as above) $\widetilde{\Xi}_{k}$ locally consists of (in general, nontransversal) smooth branches. Otherwise $P(z)$ lies in $\mathcal{D}_{k}$, which is the boundary of $\widetilde{\Xi}_{k}$, and local branches of $\widetilde{\Xi}_{k}$ near $P(z)$ might have boundary singularities. 
Let $\Omega$ be an open subset of $\mathbb{R}^{2}$ and assume that the coefficients of the recurrence relation (1.6) are sufficiently generic. The arguments in the proof of Proposition 3 applied to the induced maxmod-discriminant $\Xi_{\widetilde{\phi}}$ of the associated symbol equation (1.7) imply that the only possible singularities on the curve $\Xi_{\widetilde{\phi}}$ are the endpoints and "Y-type" singularities, i.e., triple (local) rays emanating from a given point, see Figures 1-5. Note however that the transversal intersection of smooth branches (that is, the interval and the circle) on Figure 4 is actually unstable since it disappears under a small perturbation of the coefficients of the recurrence relation.

Given a topological space $X$ let $\widehat{X}$ be its one-point compactification.

Proposition 4. The following properties hold for any $k \in \mathbb{N}$ :

(i) The hypersurfaces $\widetilde{\Xi}_{k}$ and $\Xi_{k}$ are contractible in Pol $_{k}$, and, therefore, their usual (co)homology groups are trivial.

(ii) The one-point compactification $\widehat{\Xi}_{k}$ is Alexander dual in the sphere $S^{2 k}$ to a circle $S^{1}$. Therefore, $\widehat{\Xi}_{k}$ is homotopically equivalent to a sphere $S^{2 k-2}$, so that $H_{i}\left(\widehat{\Xi}_{k}, \mathbb{Z}\right) \cong \mathbb{Z}$ if $i \in\{0,2 k-2\}$ and $H_{i}\left(\widehat{\Xi}_{k}, \mathbb{Z}\right)=0$ otherwise.

(iii) The one-point compactification $\widehat{\Xi}_{k}$ is Alexander dual in the sphere $S^{2 k}$ to $a(k-1)$-dimensional torus $\mathcal{T}^{k-1}$. Hence $\widetilde{H}_{i}\left(\widehat{\widetilde{\Xi}}_{k}, \mathbb{Z}\right) \cong \widetilde{H}^{2 k-i-1}\left(\mathcal{T}^{k-1}, \mathbb{Z}\right)$ for any $i$. Here $\widetilde{H}_{i}(X)$ (respectively, $\widetilde{H}^{i}(X)$ ) stands for reduced mod point homology (respectively, cohomology) of the topological space $X$, see e.g. [23].

Proof. The contractibility of $\widetilde{\Xi}_{k}$ and $\Xi_{k}$ follows directly from their quasihomogeneity. In order to show (ii) and (iii) we use the standard Alexander duality in $\operatorname{Pol}_{k}$. Let $\Upsilon_{k}=P o l_{k} \backslash \Xi_{k}$ and $\widetilde{\Upsilon}_{k}=P o l_{k} \backslash \widetilde{\Xi}_{k}$ denote the complements in Pol $_{k}$ of $\Xi_{k}$ and $\widetilde{\Xi}_{k}$, respectively. Then

$$
\widetilde{H}^{2 k-i}\left(\Upsilon_{k}, \mathbb{Z}\right) \cong \widetilde{H}_{i}\left(\widehat{\Xi}_{k}, \mathbb{Z}\right) \text { and } \widetilde{H}^{2 k-i}\left(\widetilde{\Upsilon}_{k}, \mathbb{Z}\right) \cong \widetilde{H}_{i}\left(\widehat{\widetilde{\Xi}}_{k}, \mathbb{Z}\right) .
$$

Obviously, the space $\widetilde{\Upsilon}_{1} \simeq \Upsilon_{1} \simeq \mathbb{C}$ is contractible. The next lemma describes the topology of $\Upsilon_{k}$ and $\widetilde{\Upsilon}_{k}$ for $k>1$.

Lemma 9. For any $k \in \mathbb{N}, k \geq 2$, one has

(i) $\widetilde{\Upsilon}_{k}$ is an open $2 k$-dimensional manifold which is homotopically equivalent to the $(k-1)$-dimensional torus $\mathcal{T}^{k-1}$.

(ii) $\Upsilon_{k}$ is an open $2 k$-dimensional manifold $\Upsilon_{k}$ which is homotopically equivalent to a circle $S^{1}$.

Proof. (i) The space $\widetilde{\Upsilon}_{k}$ consists of all $k$-tuples of complex numbers with distinct absolute values. Let $X_{k}=\left\{r_{1}<r_{2}<\ldots<r_{k} \mid r_{1} \geq 0\right\}$ denote the set of all possible $k$-tuples of distinct absolute values. Then $\widetilde{\Upsilon}_{k}$ is "fibered" over $X_{k}$ with a "fiber" which is isomorphic to $\mathcal{T}^{k}$ if $r_{1} \neq 0$ and isomorphic to $\mathcal{T}^{k-1}$ if $r_{1}=0$. In order to get an actual fibration consider the set $\widehat{X}_{k}=\left\{0<r_{2}<\ldots<r_{k}\right\}$ of the absolute values of the roots starting from the second smallest. Now $\widetilde{\Upsilon}_{k}$ is actually fibered over $\widehat{X}_{k}$ with a fiber isomorphic to $\mathcal{T}^{k-1} \times D_{r_{2}}$, where $D_{r_{2}}$ stands for the open disk of radius $r_{2}>0$ centered at the origin. The observation that $\widehat{X}_{k}$ is contractible now implies that $\widetilde{\Upsilon}_{k}$ is homotopically equivalent to $\mathcal{T}^{k-1}$.

(ii) The space $\Upsilon_{k}$ consists of all $k$-tuples of complex numbers such there exists a unique number with largest absolute value in the considered $k$-tuple. Let $0<r_{\max }$ denote this largest absolute value. Then $\Upsilon_{k}$ is fibered over $\mathbb{R}^{+} \simeq\left\{r_{\max }\right\}$ with a fiber given by the product $S^{1} \times \operatorname{Pol}_{k-1}\left(r_{\max }\right)$, where $\operatorname{Pol}_{k-1}\left(r_{\max }\right)$ stands for the set of all polynomials of degree $k-1$ whose roots lie in the open disk of radius $r_{\max }$ centered at the origin. Since both $\mathbb{R}^{+}$and $P_{0 l} l_{k-1}\left(r_{\max }\right)$ are contractible it follows that the space $\Upsilon_{k}$ is homotopically equivalent to $S^{1}$. 
Lemma 9 and the fact that $S^{1}$ is always unknotted in $P_{k}$ for $k \geq 2$ complete the proof of Proposition 4.

\section{REFERENCES}

[1] C. Berenstein, R. Gay, Complex variables. An introduction. Grad. Texts in Math., 125, Springer-Verlag, New York, 1991.

[2] J. Borcea, R. Bogvad, B. Shapiro, On rational approximation of algebraic functions, Adv. Math. 204(2) (2006), 448-480.

[3] L. Bryzgalova, The maximum functions of a family of functions that depend on parameters, Funct. Anal. Appl. 12 (1978), 66-67.

[4] E. Coussement, J. Coussement, W. Van Assche, Asymptotic zero distribution for a class of multiple orthogonal polynomials.Trans. Amer. Math. Soc. 360 (2008), no. 10, 5571-5588.

[5] S. Friedland, Invariant measures of groups of homeomorphisms and Auslander's conjecture, J. Ergod. Th. \& Dynam. Sys. 15 (1995), 1075-1089.

[6] S. Friedland, Convergence of products of matrices in projective spaces, Linear Alg. Appl. 413 (2006), 247-263.

[7] P. Griffiths and J. Harris, Principles of Algebraic Geometry, Wiley Interscience, 1978.

[8] M. Golubitsky, V. Guillemin, Stable mappings and their singularities. Grad. Texts in Math., 14, Springer-Verlag, New York-Heidelberg, 1973.

[9] L. Hörmander, The analysis of linear partial differential operators. I. Distribution theory and Fourier analysis. Reprint of the second (1990) edition. Classics in Mathematics. SpringerVerlag, Berlin, 2003.

[10] M. E. Ismail, D. R. Masson, Generalized orthogonality and continued fractions, J. Approx. Theory 83 (1995), 1-40.

[11] R.-J. Kooman, An asymptotic formula for solutions of linear second-order difference equations with regularly behaving coefficients. J. Difference Equ. Appl. 13(11) (2007), 1037-1049.

[12] R.-J. Kooman, Asymptotic behaviour of solutions of linear recurrences and sequences of Mbius-transformations. J. Approx. Theory 93(1) (1998), 1-58.

[13] V. P. Kostov, On the stratification and singularities of Stokes' hypersurface of one- and twoparameter families of polynomials, in "Theory of singularities and its applications", 251-271, Adv. Soviet Math. 1, Amer. Math. Soc., Providence, RI, 1990.

[14] A. Máté, P. Nevai, A generalization of Poincaré's theorem for recurrence equations. J. Approx. Theory 63(1) (1990), 92-97.

[15] Matov, V. I. Functions of the extremum of finite families of convex homogeneous functions. (Russian) Funktsional. Anal. i Prilozhen. 21(1) (1987), 51-62.

[16] H. Matsunaga, S. Murakami, Asymptotic behavior of solutions of functional difference equations. J. Math. Anal. Appl. 305 (2005), no. 2, 391-410.

[17] O. Perron, Über Summengleichungen und Poincarésche Differenzengleichungen, Math. Ann. 84 (1921), 1-15.

[18] H. Poincare, Sur les Equations Lineaires aux Differentielles Ordinaires et aux Differences Finies. (French) Amer. J. Math. 7(3) (1885), 203-258.

[19] M. Pituk, More on Poincarés and Perrons theorems for difference equations. J. Difference Equ. Appl. 8 (2002), no. 3, 201-216.

[20] R. Stanley, Enumerative Combinatorics, Vol. 1, Wadsworth Brooks/Cole Math. Ser., The Wadsworth \& Brooks/Cole Advanced Books \& Software, Monterey, CA, 1986.

[21] H. Stahl, Spurious poles in Páde approximation, J. Comp. Appl. Math. 99 (1998), 511-527.

[22] A. K. Tsikh, Multidimensional residues and their applications. Translated from the 1988 Russian original by E. J. F. Primrose. Translations of Mathematical Monographs, 103. American Mathematical Society, Providence, RI, 1992. x+188 pp.

[23] V. Vassiliev, Introduction to topology. Stud. Math. Libr., Vol. 14, Amer. Math. Soc., Providence, RI, 2001.

[24] H. A. van der Vorst, Computational Methods for Large Eigenvalue Problems, Handbook of numerical analysis, Vol. VIII, 3179, Handb. Numer. Anal., VIII, North-Holland, Amsterdam, 2002.

[25] A. Zhedanov, Biorthogonal rational functions and the generalized eigenvalue problem, J. Approx. Theory 101 (1999), 303-329. 
Department of Mathematics, Statistics and Computer Science, University of Illinois at Chicago, Chicago, Illinois 60607-7045

E-mail address: friedlan@uic.edu

Department of Mathematics, Stockholm University, SE-106 91 Stockholm, Sweden E-mail address: shapiro@math.su.se 